Journal of

Applied Crystallography

ISSN 0021-8898

Received 30 September 2000

Accepted 4 May 2001
(C) 2001 International Union of Crystallography Printed in Great Britain - all rights reserved

\section{Outcomes of the International Union of Crystallography Commission on Powder Diffraction Round Robin on Quantitative Phase Analysis: samples $1 \mathrm{a}$ to $1 \mathrm{~h}$}

\author{
Ian C. Madsen, ${ }^{a *}$ Nicola V. Y. Scarlett, ${ }^{a}$ Lachlan M. D. Cranswick ${ }^{b}$ and Thaung \\ Lwin $^{\mathrm{c}}$ \\ ${ }^{a}$ CSIRO Minerals, Box 312 Clayton South 3169, Victoria, Australia, ${ }^{\mathbf{b}}$ Daresbury Laboratory, \\ Warrington WA4 4AD, England, and ${ }^{\mathrm{c}} \mathrm{CSIRO}$ Mathematical and Information Sciences, Private Bag \\ 10, South Clayton MDC 3169, Victoria, Australia. Correspondence e-mail: \\ ian.madsen@minerals.csiro.au
}

The International Union of Crystallography (IUCr) Commission on Powder Diffraction (CPD) has sponsored a round robin on the determination of quantitative phase abundance from diffraction data. Specifically, the aims of the round robin were (i) to document the methods and strategies commonly employed in quantitative phase analysis (QPA), especially those involving powder diffraction, (ii) to assess levels of accuracy, precision and lower limits of detection, (iii) to identify specific problem areas and develop practical solutions, (iv) to formulate recommended procedures for QPA using diffraction data, and (v) to create a standard set of samples for future reference. Some of the analytical issues which have been addressed include $(a)$ the type of analysis (integrated intensities or full-profile, Rietveld or full-profile, database of observed patterns) and $(b)$ the type of instrument used, including geometry and radiation (X-ray, neutron or synchrotron). While the samples used in the round robin covered a wide range of analytical complexity, this paper reports the results for only the sample 1 mixtures. Sample 1 is a simple three-phase system prepared with eight different compositions covering a wide range of abundance for each phase. The component phases were chosen to minimize sample-related problems, such as the degree of crystallinity, preferred orientation and microabsorption. However, these were still issues that needed to be addressed by the analysts. The results returned indicate a great deal of variation in the ability of the participating laboratories to perform QPA of this simple threecomponent system. These differences result from such problems as (i) use of unsuitable reference intensity ratios, (ii) errors in whole-pattern refinement software operation and in interpretation of results, (iii) operator errors in the use of the Rietveld method, often arising from a lack of crystallographic understanding, and (iv) application of excessive microabsorption correction. Another major area for concern is the calculation of errors in phase abundance determination, with wide variations in reported values between participants. Few details of methodology used to derive these errors were supplied and many participants provided no measure of error at all.

\section{Introduction}

In 1996, the Commission on Powder Diffraction (CPD) of the International Union of Crystallography (IUCr) undertook a comparison of laboratories and methods in the field of quantitative phase abundance determination from diffraction data. A three-phase mixture of varying proportions was proposed as the basis of this comparison. The purpose of the study was to determine the level of precision and accuracy of the quanti- tative phase determinations of the three components in terms of the variation due to $(a)$ pure sampling and testing errors within a typical laboratory and $(b)$ differences in the analytical procedures between laboratories.

The sample 1 suite was designed to provide a relatively simple analytical system in order to determine the levels of accuracy and precision that could be expected under 'ideal' conditions. The key design criteria required that the phases exhibit little peak overlap in the low-angle region of the 
diffraction pattern and have at least one free standing peak for each phase in the $d$-spacing range 3.7-1.9 $\AA$. This ensured that participants using integrated peak methods would not have any ambiguity in the assignment of intensities arising from overlapping peaks. The phases selected needed to be crystallographically well defined materials that were readily available as high-purity powders. In addition, the materials needed to be (i) highly crystalline, to minimize the effects of peak broadening, (ii) available as finely divided powders, ensuring minimal problems with microabsorption, (iii) stable over an extended period of time, and (iv) available at a relatively low cost. While a large number of combinations of phases were considered during experiment design, the three phases selected using the criteria above were corundum $\left(\alpha-\mathrm{Al}_{2} \mathrm{O}_{3}\right)$, fluorite $\left(\mathrm{CaF}_{2}\right)$ and zincite $(\mathrm{ZnO})$. Fig. 1 shows a typical X-ray diffraction plot for sample $1 e$, where the three components are

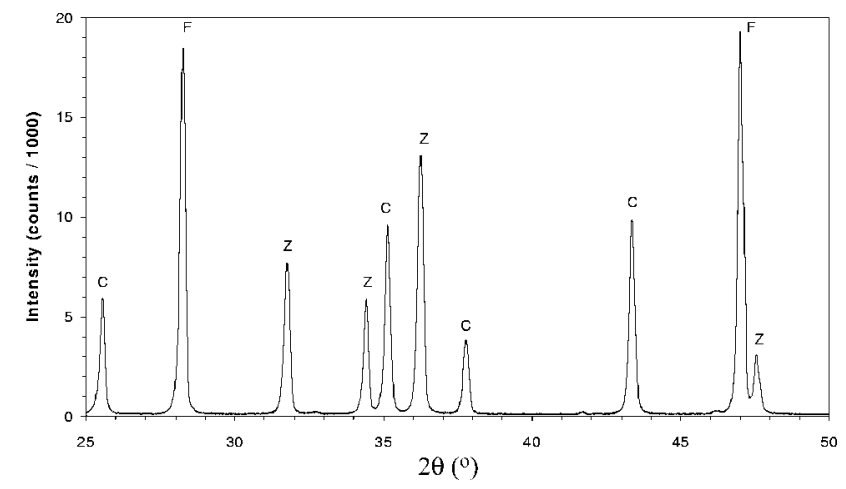

Figure 1

X-ray diffraction $(\mathrm{Cu} K \alpha)$ plot for sample 1e, where the three components are present in such proportions as to produce similar maximum intensities for all phases. The peak labels $\mathrm{C}, \mathrm{F}$ and $\mathrm{Z}$ refer to corundum, fluorite and zincite, respectively.

Table 1

Phase content of the eight mixtures comprising sample 1 derived from the weighed amounts, the elemental analyses determined by X-ray fluorescence (XRF) and quantitative phase analysis (QPA) using Rietveld analysis methods.

QPA \#1 refers to the use of the SR5 program derived from LHPM1 of Hill \& Howard (1986), while QPA \#2 refers to the KoalaRiet program described by Coelho et al. (1997). QPA \#3 refers to the use of the standardless method of Knudsen (1981). In all cases, no microabsorption correction was applied. For XRF, QPA \#1, QPA \#2 and QPA \#3, the values reported represent the mean of three separate determinations, while the e.s.d.'s are the estimated standard deviations of the means.

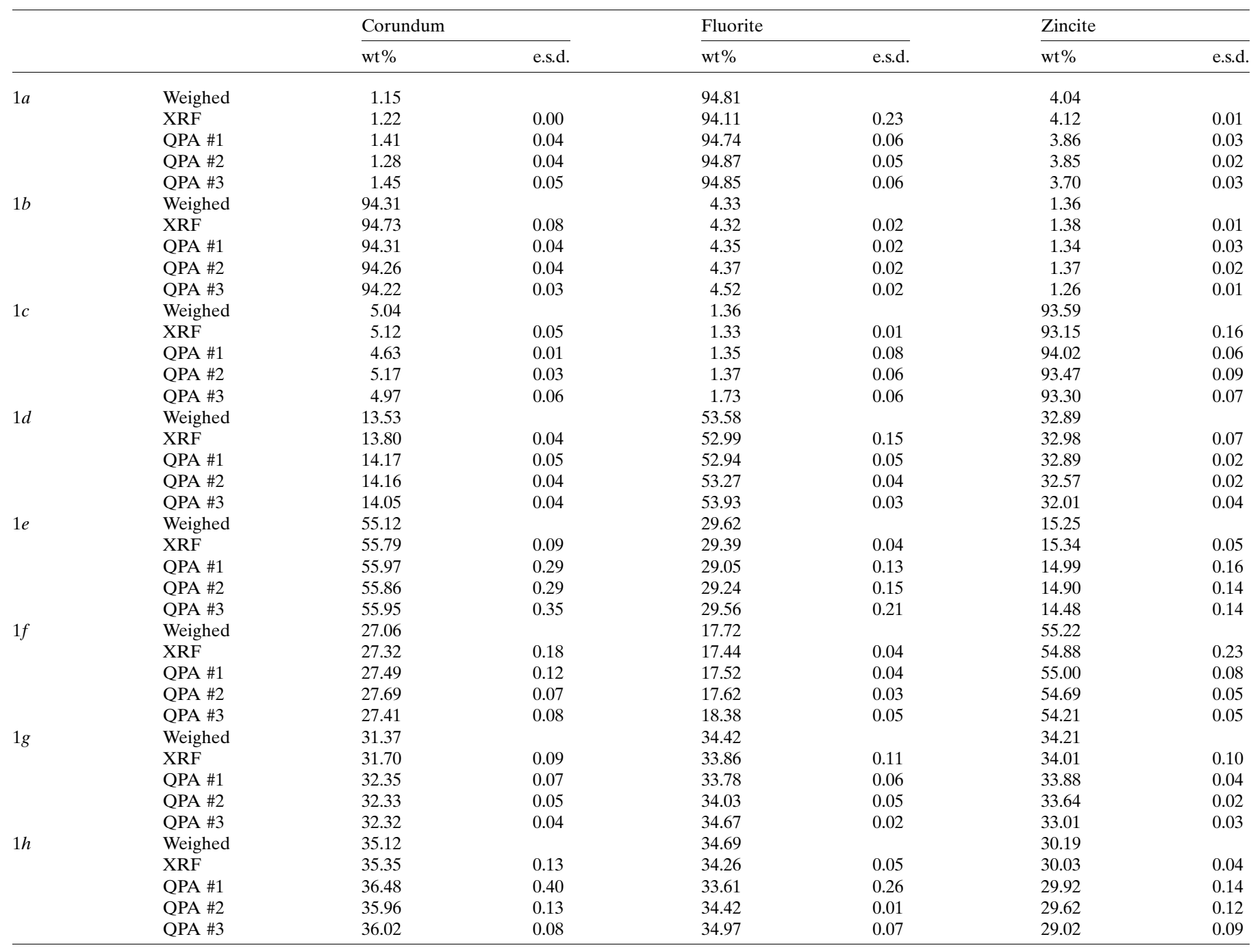




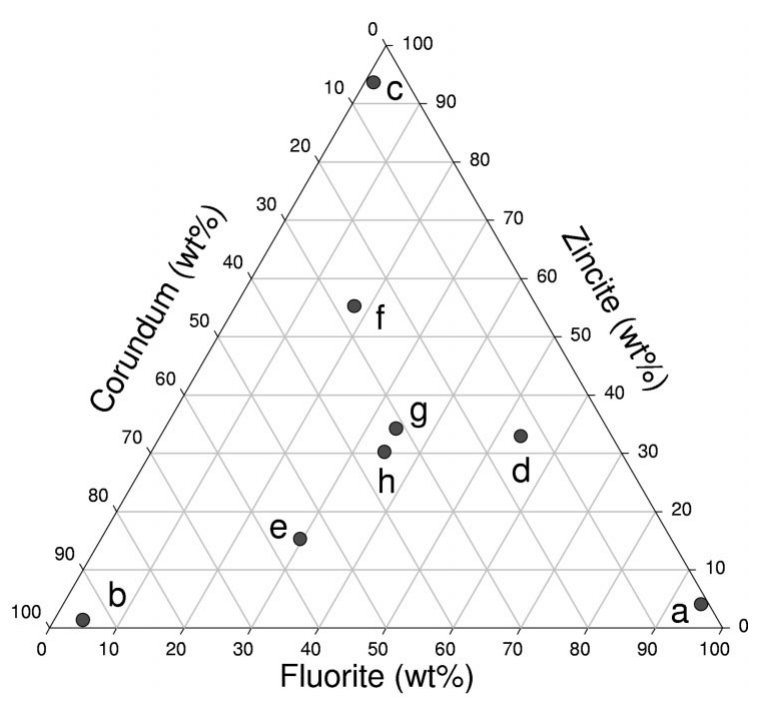

Figure 2

Ternary diagram showing the composition range of the mixtures comprising the sample 1 suite.

present in such proportions as to produce similar maximum intensities for all phases.

The three components were prepared in a ternary pattern based on a modification of a simplex design, with the design points chosen to minimize recognition by guessing. A total of eight different mixtures were included in order to cover as wide a range of composition as possible for each phase. Table 1 and Fig. 2 show that each phase is present in the sample 1 suite with concentrations of approximately 1, 4, 15, 30, 55 and $95 \mathrm{wt} \%$. Two of the mixtures $(1 g$ and $1 h)$ were prepared with very similar compositions in order to test the reproducibility within a single laboratory.

The remaining samples in the round robin address specific analytical issues in QPA, including preferred orientation (sample 2), amorphous content (sample 3) and microabsorption (sample 4). Additional samples of synthetic bauxite, natural granodiorite and synthetic pharmaceuticals served to test QPA methodology on complex 'real world' materials. The results for the remaining samples will be the subject of a separate publication.

The level of involvement of participants in the round robin was made as flexible as possible to ensure a maximum return of results to the organizers. The possible levels of participation included $(a)$ analysis of CPD-supplied 'standard' data sets by the participant and return of details of the analytical procedure along with the results, and (b) for CPD-supplied samples, analysis of the participant's own data and return of details of data collection strategies and the analytical procedure plus the results. For the sample 1 suite, all participants were required to analyse mixture $1 g$, but had the option of analysing all eight mixtures.

The CPD did not specify any details of how the analyses were to be conducted. Details of sample preparation, data collection and analysis were left to the discretion of the participant. Thus, the outcomes of the round robin also

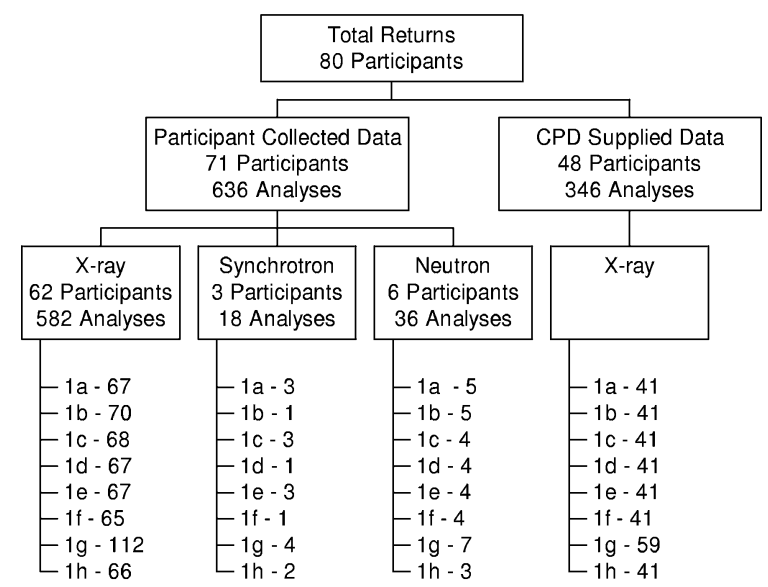

Figure 3

Summary of returns for CPD-supplied and participant-collected data partitioned according to the type of radiation used. Unless otherwise indicated, the numbers represent the number of analyses in each group.

provide a survey of current analytical practice in the determination of quantitative phase abundance from diffraction data.

A total of 128 packages of samples and accompanying 'standard' data sets and structural information were sent out in January 1998. The contents of the packages varied depending on which samples the participant agreed to analyse. A list of the participants in the round robin can be found in Table $2 ;^{\mathbf{1}}$ details of their returns are given in Fig. 3.

\section{Experimental}

\subsection{Sample preparation}

The samples were prepared from commercially available quantities of $\alpha-\mathrm{Al}_{2} \mathrm{O}_{3}$ (corundum; Baikalox Alumina Polishing Powder, standard C, $1.0 \mu \mathrm{m}$, lot $1245 \mathrm{p}$ from the Baikowski International Corporation), $\mathrm{CaF}_{2}$ (fluorite; BDH-GPR Extra Pure $\mathrm{CaF}_{2}$, product number 275944X, lot F226178 716) and $\mathrm{ZnO}$ (zincite; BDH Analar, product number 102984P, lot K21001609). Independent analyses of these materials (Stalick, 1998) showed that the corundum, fluorite and zincite were approximately 99, 100 and $100 \%$ crystalline, respectively. Inhouse measurement of particle size by Malvern Mastersizer laser sizing determined the values of particle size (based on the volume-weighted mean criteria $D[4,3]^{2}$ ) for corundum, fluorite and zincite to be $28,3.6$ and $1.4 \mu \mathrm{m}$, respectively.

For each sample, appropriate amounts of material were weighed into a beaker and flooded with petroleum spirit. Mixing was then performed using a hand blender (operating at 20000 revolutions $\mathrm{min}^{-1}$ ) for approximately $10 \mathrm{~min}$. Since petroleum spirit is a low-viscosity fluid, settling time after mixing is very short, resulting in minimal separation of phases. The mixture was filtered and dried at $383 \mathrm{~K}$ to remove excess

\footnotetext{
1 Table 2 is available in the online version of this article and from the IUCr electronic archives (Reference: HW0085). Services for accessing these data are described at the back of the journal.

${ }^{2} D[4,3]=\sum V_{i} d_{i} / \sum V_{i}$, where $V_{i}$ is the relative volume in group $i$ and $d_{i}$ is the mean group diameter.
} 
petroleum spirit and the dried filter cake passed through a coarse sieve to break up any agglomerates. Quantities of $3 \mathrm{~g}$ and $10 \mathrm{~g}$ of material were weighed into plastic vials for distribution to X-ray and neutron diffractionists, respectively.

2.1.1. Verification of mixing. To ensure that the sample mixing had been effective and that the sieving had not caused any phase segregation, X-ray diffraction data $(\mathrm{Cu} K \alpha)$ were collected from three randomly chosen portions of each sample. The data were analysed using the Rietveld (1969) analysis programs SR5 (Hill \& Howard, 1986) and KoalaRiet (Coelho et al., 1997). In addition, individual peak intensities were extracted from the data sets and analysed using the standardless method described by Knudsen (1981). To provide further confirmation, three additional randomly chosen portions were analysed by X-ray fluorescence spectrometry, in order to estimate the phase abundances from the chemical analyses. The results, given in Table 1, show that for all eight mixtures, the variation in determined phase abundances is typically less than about $0.5 \%$ relative for most concentrations, rising to 3 to $4 \%$ relative for phases present at the $1 \mathrm{wt} \%$ level.

\section{Results}

\subsection{Summary of participant methodology}

From a total of 128 packages sent out, 71 participants collected and analysed their own diffraction data, while 48 participants analysed the data supplied by the CPD, with considerable overlap between these two groups. In addition, a number of participants supplied multiple analyses using different data collection and/or analysis methods (see Fig. 3). For sample $1 g$ (the only mixture deemed mandatory by the round-robin organizers), this resulted in a total of 123 analyses of participant-collected data and 59 analyses of CPD-supplied data. For all eight mixtures comprising the sample 1 suite ( $1 a$ to $1 h$ ), these figures increase to 636 and 346 , respectively.

Many participants returned multiple analyses consisting of (i) direct replication of data collection conditions and analysis methods, (ii) collection of data using different instruments and/or geometry, and (iii) analysis of single diffraction patterns using different software and/or phase-related corrections. While the largest number of determinations per sample returned by an individual participant was eight, most participants returned one or two values. Each analysis has been considered as a separate determination in its own right. This does not account for any variations arising from data recollection procedures (i.e. sample packing, data collection time, etc.) as in most cases participants have not specified whether or not the sample was repacked and the data recollected for each new determination. In the case of multiple determinations on the CPD-supplied data, sample packing is not an issue since only one data set was supplied for each mixture. Therefore, no conclusions regarding the effect of repacking on quantitative analysis have been drawn.

3.1.1. Instrument type. All data distributed by the CPD were collected using a Philips X'Pert X-ray diffractometer fitted with a copper long-fine-focus tube operated at $40 \mathrm{kV}$ and $40 \mathrm{~mA}$. The beam path was defined using $1^{\circ}$ divergence, $0.3 \mathrm{~mm}$ receiving and $1^{\circ}$ scatter slits. A curved graphite postdiffraction monochromator was used to eliminate unwanted wavelengths. The data were collected from 15 to $145^{\circ} 2 \theta$ in steps of $0.02^{\circ}$ using a counting time of $3 \mathrm{~s} \mathrm{step}^{-1}$.

For participants who collected their own diffraction data, the majority of the returns (Fig. 3) were from X-ray diffraction users, with a small number of responses from neutron and synchrotron users. Of the X-ray participants, the vast majority used $\mathrm{Cu} K \alpha$ radiation, while a smaller group used $\mathrm{Co} K \alpha$, with one participant using Fe $K \alpha$ and two who did not specify the wavelength used. The X-ray diffraction instrument geometries consisted largely of 'conventional' Bragg-Brentano instruments, with smaller numbers of transmission, capillary and fixed sample-to-incident-beam configurations. For the neutron participants, the instruments used consisted of fixed-wavelength (with $\lambda$ ranging from 1.33 to $1.54 \AA$ ) or time-of-flight (collecting a ' $d$ ' spacing range from $0.47-6.3 \AA$ ) design with capillary sample geometry. The synchrotron participants invariably used fixed wavelengths (with $\lambda$ ranging from 0.41 to $1.15 \AA$ ) with capillary sample geometry. Fig. 4 provides a breakdown of the instrument types used and the number of participants in each group.

3.1.2. Analysis methods. For diffraction data collected by participants and supplied by the CPD, the vast majority of participants elected to conduct their analyses using a Rietveldbased method, with as many as 12 different Rietveld analysis programs used. One participant employed non-Rietveld whole-pattern matching, while the remainder used 'conventional' reference intensity ratio (RIR), iterative least squares (ILS) and internal-standard techniques. The 'internalstandard method' has been considered separately from other RIR methods when the participant has physically added an additional phase to act as an internal standard in the sample

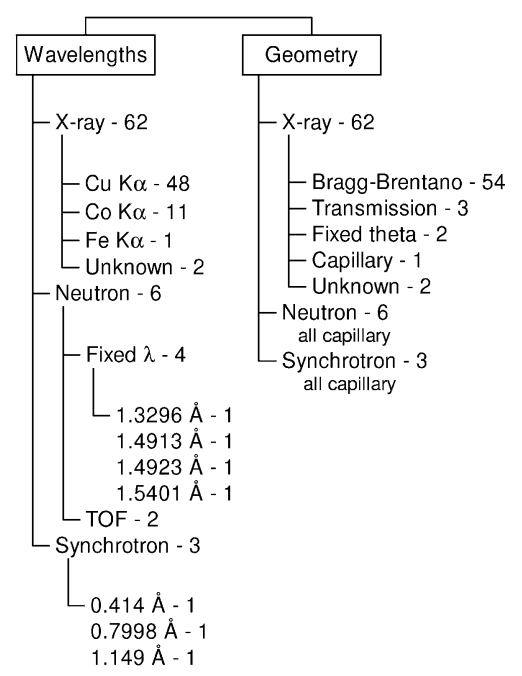

Figure 4

Summary of returns partitioned according to the wavelength and instrument geometry used for data collected by participants for sample $1 g$. The number after each entry represents the number of participants in each group. 


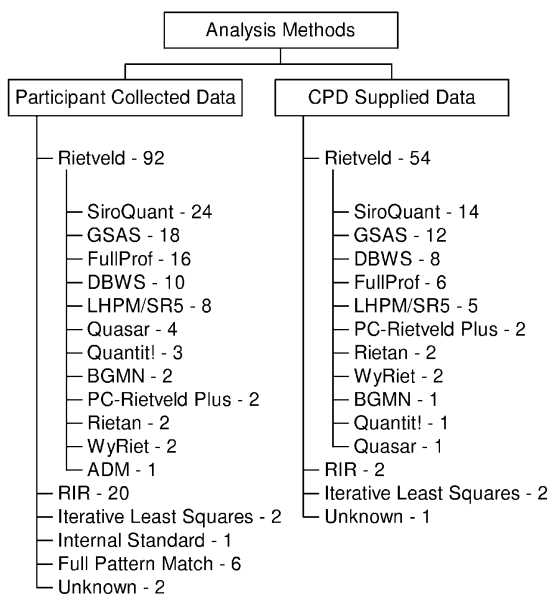

Figure 5

Summary of returns showing the analytical methods and, for the Rietveld method, the software used for analysis of data collected by participants and supplied by the CPD, for sample $1 g$ only. The number after each entry represents the number of analyses (including replicates) in each group.

prior to data collection. Fig. 5 shows the breakdown of analytical methods used in terms of data examined (participant-collected or CPD-supplied) and the total number of analyses, including replicates, contributing to each group.

3.1.3. Microabsorption correction. During data analysis, the majority of participants elected not to apply any correction for the contrast in absorption between the phases. It was stated at the start of the round robin that the phases in this sample would consist of materials with minimal phase contrast, small particle sizes and mixed to ensure homogeneity. Since the phases do exhibit a small amount of absorption contrast (Table 3), it is inevitable that a small microabsorption effect is present. Approximately one third of the results returned included a correction for microabsorption, with the majority of those participants electing to use the Brindley model.

Although the values of particle size supplied by the CPD for corundum, fluorite and zincite were 28, 3.6 and $1.4 \mu \mathrm{m}$, respectively, participants have elected to use values that range from 0.5 to $28 \mu \mathrm{m}$ for corundum, 0.5 to $13 \mu \mathrm{m}$ for fluorite and 0.5 to $11.6 \mu \mathrm{m}$ for zincite. This has resulted in large differences in the corrections applied to the determined phase abundances (see Fig. 6 for details). Note that Fig. 6 is a survey based only upon the returns given for sample $1 g$, as that was the only sample tackled by all participants. The methods described as

\section{Table 3}

Mass absorption coefficients (MAC) for each of the phases used in samples $1 a$ to $1 g$ at commonly used wavelengths.

\begin{tabular}{llccc}
\hline Tube target & Wavelength $(\AA)$ & Corundum & Fluorite & Zincite \\
\hline $\mathrm{Ti}$ & 2.749 & 169.8 & 452.8 & 245.6 \\
$\mathrm{Cr}$ & 2.290 & 100.3 & 28.07 & 148.2 \\
$\mathrm{Fe}$ & 1.936 & 61.5 & 179.0 & 93.0 \\
$\mathrm{Co}$ & 1.789 & 48.8 & 144.4 & 74.7 \\
$\mathrm{Cu}$ & 1.541 & 31.4 & 95.6 & 49.3 \\
$\mathrm{Mo}$ & 0.709 & 3.2 & 10.6 & 43.8 \\
$\mathrm{Ag}$ & 0.559 & 1.6 & 5.3 & 22.9 \\
\hline
\end{tabular}

\section{Figure 6}

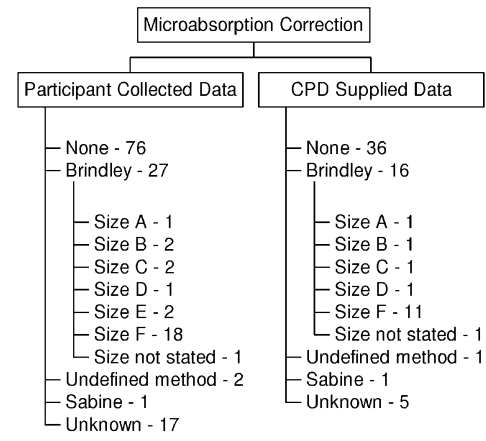

Summary of returns showing the type of microabsorption correction applied during the analysis of data collected by participants and supplied by the CPD for sample $1 g$. The number after each entry represents the number of analyses (including replicates) in each group. This figure more accurately represents the range of data analysed since many participants conducted analyses both with and without microabsorption correction. For the Brindley correction model, the assumed particle sizes $(\mu \mathrm{m})$ for the three phases, corundum, fluorite and zincite, are respectively: Size $A$ : $0.5,0.5,0.5$; Size $B: 5,5,5$; Size $C: 8.4,13,11.6$; Size $D: 10,10,10$; Size $E$ : 18, 3.6, 1.4; Size $F: 28,3.6,1.4$.

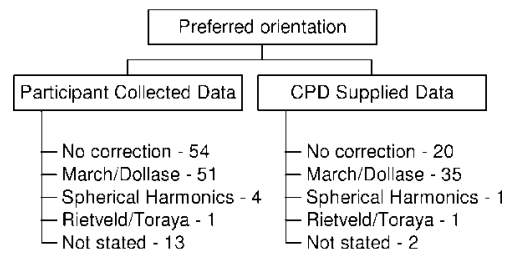

Figure 7

Summary of returns showing the type of preferred-orientation correction applied during the analysis of data collected by participants and supplied by the CPD for sample $1 g$. The number after each entry represents the number of analyses (including replicates) in each group.

'Sabine', 'Combination model' and 'Own method' will be discussed in more detail in $\$ 3.4 .3$, dealing with the results of microabsorption correction.

3.1.4. Preferred-orientation correction. The three phases used to prepare sample 1 exhibit little preferred orientation, so only minor corrections, if any, were needed to correct for any misrepresentation of peak intensity arising from nonrandom orientation of the grains. Approximately half of the analyses returned had no preferred-orientation correction applied while, for those analyses where a correction had been applied, the March-Dollase model was the most commonly used (Fig. 7).

\subsection{Summary of returned results}

Table 4 provides a summary of all the returned results for data supplied by the CPD and for data collected by participants. The summary shows that, while the mean values for each group approximate the weighed values, there is a very wide range of values within the group, evidenced by the large values for the group standard deviations. Individual results for all samples are also presented graphically in Figs. 8 to $15^{\mathbf{3}}$

\footnotetext{
${ }^{3}$ Figs. 8 to 15 are available in the online version of this article and from the IUCr electronic archives (Reference: HW0085). Services for accessing these data are described at the back of the journal.
} 
Preliminary examination of the results indicates the following.

(a) In general, inaccuracies and errors are far greater for the analysis of participant-collected data than for the analysis of CPD-supplied data.

(b) Some $70 \%$ of participants have provided an estimate of the error of determination for each analysis, but the magnitude of these estimates varies widely between participants. For example, for sample $1 g$, the reported errors in the corundum estimates vary from 0.0 to $5.5 \mathrm{wt} \%$, representing a relative error ranging from 0.0 up to $18 \%$.

(c) Phases present only in minor amounts are more difficult to determine than the phases present in medium to major amounts. This is especially true for corundum, which, for X-ray radiation, has the lowest average 'scattering power' and hence the lowest observed intensities of the three phases.

\subsection{Statistical method of results analysis}

Two error estimates were used to assess the validity of the returns from participants. The absolute bias for the determination of each analysed component phase was defined as

$\begin{aligned} \operatorname{Bias}(w \mathrm{t} \%)= & \operatorname{Measured}\left(\mathrm{wt}_{0} \%\right) \\ & -\operatorname{True}\left(\mathrm{wt}^{0} \%\right)\end{aligned}$

$$
-\operatorname{True}(w \mathrm{w} \%) \text {, }
$$

where the weighed values of the components were used as the 'true' values.

The relative bias $(\mathrm{RB})$ is then defined as

$$
\mathrm{RB}=100 \times \frac{\operatorname{Bias}\left(\mathrm{wt}_{0} \%\right)}{\operatorname{True}\left(\mathrm{wt}_{0} \%\right)}
$$

The relative bias gives an indication of the accuracy of the determination of each phase within a sample. In order to assess the analysis of the sample as a whole, it was necessary to combine the individual phase assessments in some way. It was not possible to use a simple sum of relative bias values due to the fact that most Rietveld results are reported in normalized fashion, i.e. all analysed phases are summed to $100 \%$. This, in turn, makes the sum of
Table 4

Summary of the quantitative phase analyses (wt\%) derived from data supplied by the CPD and collected by the participants.

In addition, the results from participant-collected data have been divided into sub-groups determined by the type of radiation used, namely laboratory $\mathrm{X}$-ray, neutron and synchrotron radiation. The statistics include all participant determinations, including replicates. $N$ represents the total number of measurements included in the statistics for each sample. The s.d. value is the standard deviation of the mean, while min. and max. represent the minimum and maximum values in each group, respectively.

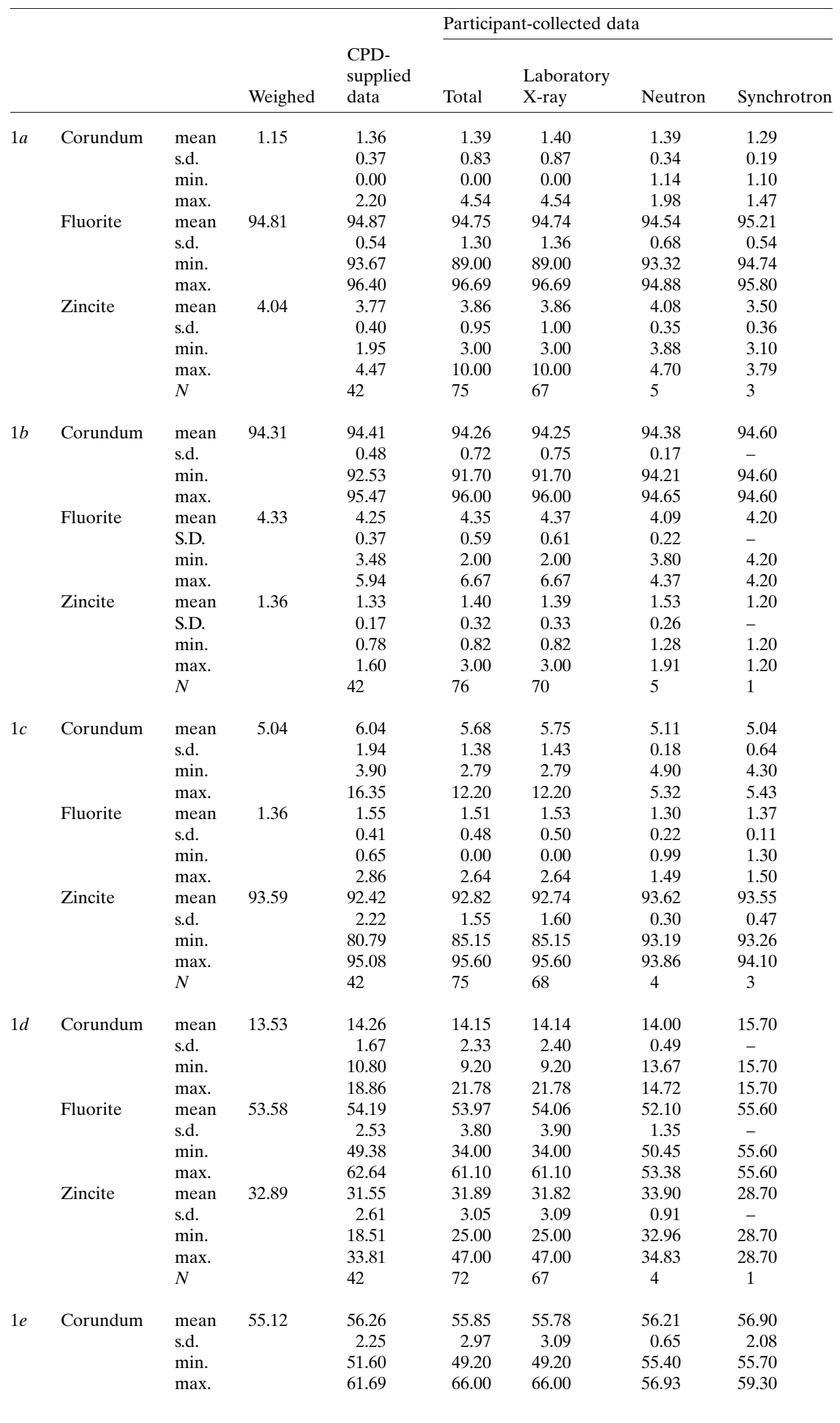


Table 4 (continued)

\begin{tabular}{|c|c|c|c|c|c|c|c|c|}
\hline & & & & & Particip & at-collected d & & \\
\hline & & & Weighed & $\begin{array}{l}\text { supplied } \\
\text { data }\end{array}$ & Total & $\begin{array}{l}\text { Laboratory } \\
\text { X-ray }\end{array}$ & Neutron & Synchrotron \\
\hline & Fluorite & mean & 29.62 & 29.55 & 29.59 & 29.68 & 28.36 & 29.23 \\
\hline & & s.d. & & 1.89 & 3.02 & 3.15 & 0.95 & 0.72 \\
\hline & & $\min$. & & 25.22 & 16.00 & 16.00 & 27.22 & 28.40 \\
\hline & & $\max$ & & 33.46 & 35.50 & 35.50 & 29.20 & 29.70 \\
\hline & Zincite & mean & 15.25 & 14.20 & 14.57 & 14.55 & 15.44 & 13.86 \\
\hline & & s.d. & & 1.34 & 1.10 & 1.10 & 0.40 & 1.35 \\
\hline & & $\min$. & & 7.68 & 11.40 & 11.40 & 14.90 & 12.30 \\
\hline & & $\max$. & & 16.10 & 18.00 & 18.00 & 15.85 & 14.68 \\
\hline & & $N$ & & 42 & 74 & 67 & 4 & 3 \\
\hline $1 f$ & Corundum & mean & 27.06 & 28.33 & 27.90 & 27.91 & 27.23 & 29.80 \\
\hline & & s.d. & & 2.85 & 2.91 & 3.00 & 0.52 & - \\
\hline & & $\min$. & & 23.00 & 20.10 & 20.10 & 26.70 & 29.80 \\
\hline & & $\max$. & & 41.20 & 35.87 & 35.87 & 27.94 & 29.80 \\
\hline & Fluorite & mean & 17.72 & 18.46 & 18.35 & 18.41 & 16.96 & 20.20 \\
\hline & & s.d. & & 1.72 & 2.27 & 2.31 & 0.73 & - \\
\hline & & $\min$. & & 15.54 & 9.00 & 9.00 & 16.08 & 20.20 \\
\hline & & $\max$. & & 24.10 & 22.91 & 22.91 & 17.60 & 20.20 \\
\hline & Zincite & mean & 55.22 & 53.21 & 53.74 & 53.68 & 55.81 & 50.00 \\
\hline & & s.d. & & 3.59 & 2.82 & 2.84 & 0.37 & - \\
\hline & & $\min$. & & 34.70 & 45.65 & 45.65 & 55.31 & 50.00 \\
\hline & & $\max$ & & 57.70 & 59.00 & 59.00 & 56.15 & 50.00 \\
\hline & & $N$ & & 41 & 70 & 65 & 4 & 1 \\
\hline $1 g$ & Corundum & mean & 31.37 & 31.55 & 32.25 & 32.24 & 32.13 & 32.83 \\
\hline & & s.d. & & 4.50 & 3.30 & 3.44 & 0.41 & 1.86 \\
\hline & & $\min$. & & 9.60 & 22.40 & 22.40 & 31.48 & 30.80 \\
\hline & & $\max$. & & 40.42 & 48.60 & 48.60 & 32.66 & 35.20 \\
\hline & Fluorite & mean & 34.42 & 35.50 & 34.81 & 34.91 & 33.26 & 34.73 \\
\hline & & s.d. & & 6.35 & 3.05 & 3.16 & 0.91 & 0.48 \\
\hline & & $\min$. & & 30.79 & 19.00 & 19.00 & 31.82 & 34.30 \\
\hline & & $\max$ & & 80.40 & 41.70 & 41.70 & 34.51 & 35.40 \\
\hline & Zincite & mean & 34.21 & 33.07 & 32.95 & 32.86 & 34.60 & 32.48 \\
\hline & & s.d. & & 5.21 & 2.31 & 2.35 & 0.61 & 2.22 \\
\hline & & $\min$. & & 10.00 & 24.70 & 24.70 & 34.01 & 29.40 \\
\hline & & $\max$ & & 59.33 & 42.00 & 42.00 & 35.51 & 34.60 \\
\hline & & $N$ & & 59 & 123 & 112 & 7 & 4 \\
\hline $1 h$ & Corundum & mean & 35.12 & 35.96 & 35.73 & 35.65 & 35.92 & 37.46 \\
\hline & & s.d. & & 2.71 & 3.47 & 3.58 & 0.86 & 2.29 \\
\hline & & $\min$. & & 30.30 & 26.80 & 26.80 & 35.04 & 36.10 \\
\hline & & $\max$ & & 43.83 & 44.00 & 44.00 & 36.76 & 40.10 \\
\hline & Fluorite & mean & 34.69 & 35.21 & 34.77 & 34.85 & 33.37 & 34.48 \\
\hline & & s.d. & & 2.40 & 4.15 & 4.31 & 1.56 & 0.13 \\
\hline & & $\min$. & & 30.33 & 16.00 & 16.00 & 32.08 & 34.34 \\
\hline & & max. & & 40.30 & 41.80 & 41.80 & 35.10 & 34.60 \\
\hline & Zincite & mean & 30.19 & 28.93 & 29.40 & 29.40 & 30.71 & 28.06 \\
\hline & & s.d. & & 2.56 & 3.80 & 3.92 & 0.73 & 2.39 \\
\hline & & $\min$. & & 16.49 & 23.90 & 23.90 & 29.87 & 25.30 \\
\hline & & $\max$. & & 33.81 & 55.90 & 55.90 & 31.15 & 29.48 \\
\hline & & $N$ & & 42 & 72 & 66 & 3 & 3 \\
\hline
\end{tabular}

in a phase present at the $1 \mathrm{wt} \%$ level is vastly different from a relative error of $10 \%$ in a phase present at the $90 \mathrm{wt} \%$ level. The KLD provides a value that can be averaged over groups of data to determine which instruments, methods or participants were able to measure the phase compositions most accurately. The most accurate values are represented by a small absolute value of KLD, while over- and underestimations return negative and positive values, respectively.

For an individual sample, the KLD for all three phases can be estimated using

$$
\begin{aligned}
\mathrm{KLD}_{\text {sum }}= & \mathrm{KLD}_{\text {corundum }}+\mathrm{KLD}_{\text {fluorite }} \\
& +\mathrm{KLD}_{\text {zincite }}
\end{aligned}
$$

Where a general estimate of the magnitude of error within an analysis was required, rather than the source of the error, absolute values of the Kullback-Leibler distances (AKLD) have been calculated thus:

$$
\begin{aligned}
\mathrm{AKLD}_{\text {sum }}= & \mathrm{AKLD}_{\text {corundum }} \\
& +\mathrm{AKLD}_{\text {fluorite }} \\
& +\mathrm{AKLD}_{\text {zincite }}
\end{aligned}
$$

To assess the variation of AKLD within a group, an approximate estimate of the $95 \%$ confidence limits can be obtained by first determining the standard deviation (s.d.) of all values of AKLD in the group and calculating the uncertainty according to

$$
\text { Uncertainty }=\frac{2 \times \text { s.d. of AKLD }}{N^{1 / 2}},
$$

where $N$ is the number of values in the group. This is based upon the assumption that a population of

relative biases zero. In order to overcome this problem and to provide a single numerical assessment of the performance of each group, a statistic based on the Kullback-Leibler distance (KLD) was employed. The conventional KLD equation (Kullback, 1968) has been weighted to allow for the concentration of the phase and is defined as

$$
\mathrm{KLD}=0.01 \times \operatorname{True}\left(\mathrm{wt}_{0} \%\right) \times \ln \left[\frac{\operatorname{True}\left(\mathrm{wt}_{0} \%\right)}{\operatorname{Measured}\left(\mathrm{wt}_{0} \%\right)}\right] .
$$

This weighting for concentration is most important for a true assessment of an analysis. For example, a relative error of $10 \%$ sufficient size will approximate a normal distribution. The smaller the population, the more approximate the estimate. The significance of differences between groups is based on their combined uncertainties.

For the assessment of reproducibility by an individual laboratory, the variance in the errors between samples $1 g$ and $1 h$ is calculated for each phase using

$$
\operatorname{Variance}\left[\Delta_{1 g(\text { phase } i)}, \Delta_{1 h(\text { phase } i)}\right]=\frac{\left[\Delta_{1 g(\text { phase } i)}-\Delta_{1 h(\text { phase } i)}\right]^{2}}{2},
$$


where $\Delta_{1 g \text { (phase } i)}=\left(\right.$ Measured $_{1 g}-$ True $\left._{1 g}\right)$ for phase $i$, and $\Delta_{1 h(\text { phase } i)}=\left(\right.$ Measured $_{1 h}-$ True $\left._{1 h}\right)$ for phase $i$ with the weighed values taken as the 'true' estimates.

The relative standard deviation (\%r.s.d.) for a group is then determined by averaging the variances within the group and applying the formula

$$
\% \text { r.s.d. }{ }_{\text {group }}=\frac{100 \times(\text { Average variance for group })^{1 / 2}}{0.5 \times\left(\text { True }_{1 g}+\text { True }_{1 h}\right)} .
$$

\subsection{Summary of results analysis}

3.4.1. Instrument type. The $\mathrm{AKLD}_{\text {sum }}$ has been averaged over all returned results partitioned according to the type of radiation used to collect data (Table 5).

The summary shows that results determined from neutron radiation are significantly closer to the 'true' values than participant-collected laboratory-based X-ray data. The CPDsupplied X-ray data and synchrotron results overlap both groups. The better result from the neutron users may arise predominantly from the decreased errors arising from samplerelated effects, such as microabsorption. However, it may also be contributed to by the fact that neutron users are likely to have a greater degree of expertise in the application of complex analytical methods (i.e. the Rietveld method) than many laboratory-based X-ray users. It should be noted that results derived from fixed-wavelength neutron data are significantly better than those derived from time-of-flight data. In addition, the results derived from short-wavelength (0.4135 ̊) synchrotron data are significantly better than those using longer wavelength (1.149 $\AA$ ) data, probably as a result of a decrease in any residual microabsorption effects. For laboratory-based X-ray data, there is no significant difference between results derived from $\mathrm{Cu}$ and $\mathrm{Co}$ radiation.

3.4.2. Analysis type. For the data collected and distributed by the CPD, there is no significant difference between the

\section{Table 5}

Average of the absolute values of the Kullback-Liebler distance $\left(\mathrm{AKLD}_{\text {sum }}\right)$ for all returned results partitioned according to the type of radiation used to collect data.

The values in parentheses represent the $95 \%$ confidence limits calculated using equation (6). The neutron returns have been further partitioned according to the instrument geometry, namely fixed wavelength or time of flight (TOF). Similarly, the synchrotron and laboratory X-ray returns have been partitioned according to the wavelength used.

\begin{tabular}{|c|c|c|c|c|}
\hline & \multirow{2}{*}{$\begin{array}{l}\text { CPD- } \\
\text { supplied } \\
\text { X-ray }\end{array}$} & \multicolumn{3}{|c|}{ Participant-collected data } \\
\hline & & Neutron & Synchrotron & $\begin{array}{l}\text { Laboratory } \\
\text { X-ray }\end{array}$ \\
\hline Average $\mathrm{AKLD}_{\text {sum }}$ & $0.036(6)$ & $\begin{array}{l}0.018(6)-\text { All } \\
0.012(5)-\text { Fixed } \lambda \\
0.034(12)-\text { TOF }\end{array}$ & $\begin{array}{l}0.036(17)-\text { All } \\
0.015(5)-0.4135 \AA \\
0.012(-)-0.7998 \AA \\
0.064(30)-1.149 \AA\end{array}$ & $\begin{array}{l}0.053(4)-\text { All } \\
0.048(6)-\operatorname{Cu} K \alpha \\
0.059(7)-\operatorname{Co~} K \alpha \\
0.33(-)-\text { Fe } K \alpha \\
0.052(22)-\text { Unknown }\end{array}$ \\
\hline Number of analyses & 456 & $\begin{array}{l}36-\text { All } \\
26-\text { Fixed } \lambda \\
10-\text { TOF }\end{array}$ & $\begin{array}{l}19-\text { All } \\
10-0.4135 \AA \\
1-0.7998 \AA \\
8-1.149 \AA\end{array}$ & $\begin{array}{l}582-\text { All } \\
371-\mathrm{Cu} K \alpha \\
201-\mathrm{Co} K \alpha \\
1-\mathrm{Fe} K \alpha \\
9-\text { Unknown }\end{array}$ \\
\hline
\end{tabular}

analytical methods employed by participants (Table 6). However, for data collected by participants, while there is a similar range of errors for iterative least squares (ILS), Rietveld and full-pattern analysis methodology, there are significantly larger errors for methods based on the use of RIR values and internal standards (Table 7).

3.4.3. Microabsorption correction. The analysis of phases present in these samples should exhibit minimal deviation caused by the effect of absorption contrast. However, many participants elected to apply a correction during the analysis. For both the CPD-supplied and participant-collected data, there is little difference in the group errors for the use of the Brindley correction model (Brindley, 1945) and the application of no correction at all (Tables 8 and 9; note that Tables 8 and 9 refer to all results returned). The method listed as that of Sabine (Sabine et al., 1998) refers to an incorrect application of a method for correction of absorption in capillaries. This method is not applicable to microabsorption and the returned values are significantly worse than those for no correction at all.

One participant has supplied results incorporating their own method of microabsorption correction. This method is described as an empirical correlation developed from their own data on reference mixtures. It is based upon the deviation of the absorption coefficient of the individual phase from that of the mixture. This method has returned significantly better results than no correction. Another participant has applied what they describe as a 'combination model' for correction and produced significantly better results than for no correction. The figures for these lesser known methods, however, cannot be regarded as definitive due to the size of the populations. For the participant-collected data, the neutron results have been separated out since the effects of microabsorption should be minimal. Their average values of $\mathrm{AKLD}_{\text {sum }}$ again are significantly lower than those obtained by X-ray participants.

3.4.4. Preferred-orientation correction. Again, while the three phases present in the sample 1 suite exhibit little preferred orientation, many participants have elected to apply a correction during data analysis. For the CPD-supplied data there is no significant difference between the results obtained with corrections applied and the results returned using no correction (Table 10). For the participant-collected data, there is a significant improvement in the results that incorporated a spherical harmonics correction in the refinement strategy (Table 11 ). There is no significant difference between results corrected using the MarchDollase model and those returned with no correction.

3.4.5. Reproducibility. Reproducibility in this survey has been defined 
Table 6

Average of the absolute values of the Kullback-Liebler distance $\left(\mathrm{AKLD}_{\text {sum }}\right)$ for returned results partitioned according to analytical method employed - CPD-supplied data.

The values in parenthesis represent the $95 \%$ confidence limits calculated using equation (6).

\begin{tabular}{llll}
\hline & Rietveld & RIR & ILS \\
\hline Average AKLD sum & $0.044(9)$ & $0.046(32)$ & $0.047(17)$ \\
Number of analyses & 319 & 9 & 8 \\
\hline
\end{tabular}

as the ability of a participant to analyse two separate samples

\section{Table 7}

Average of the absolute values of the Kullback-Liebler distance $\left(\mathrm{AKLD}_{\text {sum }}\right)$ for returned results partitioned according to analytical method employed - participant-collected data.

The values in parenthesis represent the $95 \%$ confidence limits calculated using equation (6).

\begin{tabular}{lllllll}
\hline & ILS & Rietveld & $\begin{array}{l}\text { Full-pattern } \\
\text { analysis }\end{array}$ & Unknown & RIR & $\begin{array}{l}\text { Internal } \\
\text { standard }\end{array}$ \\
\hline $\begin{array}{l}\text { Average AKLD } \\
\text { Number of analyses }\end{array}$ & $0.036(13)$ & $0.044(4)$ & $0.045(14)$ & $0.075(47)$ & $0.089(19)$ & $\begin{array}{l}0.101(31) \\
8\end{array}$ \\
\hline
\end{tabular}

\section{Table 8}

Average of the absolute values of the Kullback-Liebler distance $\left(\mathrm{AKLD}_{\text {sum }}\right)$ for returned results partitioned according to microabsorption method employed - CPD-supplied data.

The values in parenthesis represent the $95 \%$ confidence limits calculated using equation (6).

\begin{tabular}{llllll}
\hline & No correction & Brindley & Sabine & Unknown & Own method \\
\hline Average AKLD & $0.046(6)$ & $0.044(3)$ & $0.070(2)$ & $0.034(5)$ & $0.012(2)$ \\
Number of analyses & 218 & 105 & 2 & 19 & 8 \\
\hline
\end{tabular}

\section{Table 9}

Average of the absolute values of the Kullback-Liebler distance $\left(\mathrm{AKLD}_{\text {sum }}\right)$ for returned results partitioned according to microabsorption method employed - participant-collected data.

The values in parenthesis represent the $95 \%$ confidence limits calculated using equation (6).

\begin{tabular}{llllllll}
\hline & $\begin{array}{l}\text { No } \\
\text { correction }\end{array}$ & Neutron & Brindley & Sabine & Unknown & $\begin{array}{l}\text { Own } \\
\text { method }\end{array}$ & $\begin{array}{l}\text { Combination } \\
\text { model }\end{array}$ \\
\hline $\begin{array}{c}\text { Average } \\
\begin{array}{c}\text { AKLD } \\
\text { sum }\end{array}\end{array}$ & $0.050(3)$ & $0.018(3)$ & $0.054(4)$ & $0.091(1)$ & $0.062(5)$ & $0.022(5)$ & $0.023(5)$ \\
$\begin{array}{c}\text { Number of } \\
\text { analyses }\end{array}$ & 336 & 36 & 154 & 2 & 93 & 8 & 8 \\
\hline
\end{tabular}

\section{Table 10}

Average of the absolute values of the Kullback-Liebler distance $\left(\mathrm{AKLD}_{\text {sum }}\right)$ for returned results partitioned according to preferred-orientation method employed - CPD-supplied data.

The values in parenthesis represent the $95 \%$ confidence limits calculated using equation (6).

\begin{tabular}{llllll}
\hline & $\begin{array}{l}\text { Rietveld- } \\
\text { Toraya }\end{array}$ & $\begin{array}{l}\text { Spherical } \\
\text { harmonics }\end{array}$ & Unknown & $\begin{array}{l}\text { No } \\
\text { correction }\end{array}$ & $\begin{array}{l}\text { March- } \\
\text { Dollase }\end{array}$ \\
\hline $\begin{array}{l}\text { Average AKLD } \\
\text { Number of analyses }\end{array}$ & $0.022(9)$ & $0.024(11)$ & $0.028(11)$ & $0.040(11)$ & $0.048(11)$ \\
\hline
\end{tabular}

Table 11

Average of the absolute values of the Kullback-Liebler distance $\left(\mathrm{AKLD}_{\text {sum }}\right)$ for returned results partitioned according to preferred-orientation method employed - participant-collected data.

The values in parenthesis represent the $95 \%$ confidence limits calculated using equation (6).

\begin{tabular}{llllll}
\hline & $\begin{array}{l}\text { Rietveld- } \\
\text { Toraya }\end{array}$ & $\begin{array}{l}\text { Spherical } \\
\text { harmonics }\end{array}$ & Unknown & $\begin{array}{l}\text { No } \\
\text { correction }\end{array}$ & $\begin{array}{l}\text { March- } \\
\text { Dollase }\end{array}$ \\
\hline $\begin{array}{l}\text { Average AKLD } \\
\text { Number of analyses }\end{array}$ & $0.041(-)$ & $0.029(9)$ & $0.059(12)$ & $0.055(9)$ & $\begin{array}{l}0.047(5) \\
716\end{array}$ \\
\hline
\end{tabular}

of very similar, although not identical, composition (samples $1 g$ and $1 h)$. The exact compositions for samples $1 g$ and $1 h$ can

The determination of reproducibility has been based upon he percent relative standard deviation (\%r.s.d.) of a group is a measure of the precision of the determinations rather than their absolute accuracy, i.e. how reliably a participant can analyse samples of similar composition. Since not all participants analysed both samples $1 g$ and $1 h$, the populations in some of the groups are rather low and the reproducibility of such groups may owe more to the expertise (or otherwise) of a particular participant than the methodology in question. Tables 12 , 13 and 14 show the \%r.s.d. figures for all data separated into various groups.

Comparison of Tables 12 and 13 shows the significant improvement in reproducibility achieved when participants analyse CPD-supplied data rather than data they have collected themselves. Examining the data on the basis of analytical method, reveals that an unspecified method applied to the CPD-supplied data produced the best reproducibility. However, this is not a valid conclusion because of the group size $(n=1)$ and may be more a reflection of the skill of a particular operator than anything else. Considering the participant-collected data in groups according to data collection method (Table 14) shows (i) that there is better reproducibility for neutron data than either synchrotron or laboratory-based X-ray data, and (ii) synchrotron data yielded better reproducibility than participantcollected X-ray data. Once again, there is a distinct imbalance in the group sizes being compared, i.e. $n=3$ for both neutron and synchrotron data and $n=65$ for X-ray data. However, the group sizes of neutron and synchrotron data are directly comparable and there is an apparent improvement in reproducibility with neutron data. It is interesting to note that \%r.s.d. values obtained with synchrotron data are very similar to 
those obtained for the analysis of the CPD-supplied data, which is laboratory X-ray derived.

One curious feature, which appears to have no physical basis, is the improvement in reproducibility gained by using $\mathrm{Co} K \alpha$ radiation instead of $\mathrm{Cu} K \alpha$ radiation in conventional laboratory-based X-ray sets (Table 14). One possible explanation for this is the proliferation of $\mathrm{Cu} K \alpha$ radiation used in conventional X-ray instruments. This may mean that there are more instruments with $\mathrm{Cu}$ target tubes being used by lessexperienced operators just through sheer weight of numbers. Once again, this may merely be a reflection of operator expertise rather than any true instrumental bias, as there is little change in absorption contrast across this particular phase system when changing from $\mathrm{Cu} K \alpha$ to $\mathrm{Co} K \alpha$ radiation (Table 3).

\section{Discussion}

The types of analytical issues that have become apparent during analysis of the returns can be divided into a number of groups. While these groups do not cover every cause of error in the returns, they adequately represent the major areas for concern in QPA uncovered during this study.

\subsection{Use of inappropriate RIR values}

Several participants have used conventional 'single-peak' methods for QPA coupled with the so-called reference intensity ratios for calibration. It should be noted that RIR methods may also have been used with multiple peaks, but in this instance participants have not indicated whether this is the case or not. The spread of RIR values returned by the participants (and hence QPA results) indicates that there exists some confusion regarding the 'correct' values of RIR to use. Table 15 shows those RIR values that were supplied by participants (most were not reported), along with the corresponding phase analysis values. In addition, values of RIR published by the JCPDS-ICDD (ICDD, 1999) and by Smith $e t$ al. (1987) have been included for reference. These values have been included to highlight further the different RIR values available and the necessity of selecting those which are applicable to the particular experiment in question, i.e. appropriate to particular instrumental considerations and for use with integrated intensities or peak heights (examples of both are included in the published ICDD values). Not only is there significant variation in the RIR values employed by participants, but a similar variation also exists in the published values (generally considered as the sources of RIR values for analysts).

The values of RIR needed will vary depending on the analytical strategy used (e.g. peak height, peak area, whole pattern, X-ray wavelength), with an incorrect choice resulting in errors in the derived phase abundance. By way of example, participant $C$ (Table 15) has employed peak area measurements based on the strongest peak for each phase. An RIR value of 3.30 for fluorite taken from Smith et al. (1987) has been used in spite of the fact that this value was derived for
Table 12

Summary of the reproducibility assessments (\%r.s.d.) for the CPDsupplied data partitioned according to analysis method.

$\%$ r.s.d. is given by equation (8). $n$ is the population size.

\begin{tabular}{llll}
\hline & \%r.s.d. & & \\
\cline { 2 - 4 } Analysis method & Corundum & Fluorite & Zincite \\
\hline Rietveld $(n=38)$ & 1.1 & 0.9 & 1.6 \\
RIR $(n=1)$ & 0.5 & 0.04 & 0.5 \\
ILS $(n=2)$ & 2.1 & 1.2 & 0.9 \\
Unknown $(n=1)$ & 0.3 & 0.4 & 0.1 \\
Total $(n=42)$ & 1.2 & 0.9 & 1.6 \\
\hline
\end{tabular}

Table 13

Summary of the reproducibility assessments (\%r.s.d.) for the participantcollected data partitioned according to analysis method.

\begin{tabular}{llll}
\hline & \multicolumn{2}{l}{ \%r.s.d. } & \\
\cline { 2 - 4 } Analysis method & Corundum & Fluorite & Zincite \\
\hline Rietveld $(n=57)$ & 3.6 & 4.5 & 7.4 \\
RIR $(n=8)$ & 2.9 & 1.7 & 2.1 \\
ILS $(n=2)$ & 1.3 & 0.8 & 0.7 \\
Full-pattern match $(n=2)$ & 8.4 & 6.5 & 2.8 \\
Internal standard $(n=1)$ & 14.6 & 0.3 & 0.04 \\
Unknown $(n=1)$ & 0.5 & 4.6 & 4.4 \\
Total $(n=71)$ & 4.1 & 4.3 & 6.7 \\
\hline
\end{tabular}

Table 14

Summary of the reproducibility assessments (\%r.s.d.) for participantcollected data partitioned according to data collection method.

\begin{tabular}{llll}
\hline & \%r.s.d. & & \\
\cline { 2 - 4 } Data collection method & Corundum & Fluorite & Zincite \\
\hline Neutron $(n=3)$ & 0.49 & 0.50 & 0.56 \\
Synchrotron $(n=3)$ & 1.80 & 1.36 & 1.18 \\
X-ray (total) $(n=65)$ & 4.23 & 4.42 & 7.01 \\
X-ray $(\mathrm{Cu} K \alpha)(n=40)$ & 4.67 & 5.45 & 8.76 \\
X-ray (Co $K \alpha)(n=24)$ & 3.48 & 1.83 & 2.21 \\
XRF $(n=3)$ & 0.37 & 0.33 & 0.26 \\
\hline
\end{tabular}

use with whole-pattern analysis techniques. Published RIR values may also be inappropriate if sample-related parameters (e.g. crystallinity) of the material being analysed differ from those of the material used to derive the RIR.

Additional errors can occur in the application of these methods since they generally rely on estimation of the intensity (area or height) of a single peak rather than using all of the peaks in the pattern as in whole-pattern (e.g. Rietveld) methods. Any errors in the peak intensity measurement will be in addition to the errors in the RIR values used. Errors in peak intensity estimates can be reduced by using the multiplepeak approach of Hubbard \& Snyder (1988), but values of RIR specific to the peaks used in the analysis need to be employed. Even though no attempt has been made in this study to resolve the effect of errors in the peak intensity and the RIR values, there is sufficient ambiguity in reported and published values to suggest that further work needs to be performed in resolving differences in published RIR values and clarifying their area of application. 


\subsection{Excessive microabsorption correction}

Many analysis programs have corrections for absorption contrast built in and operators often apply the correction without knowing whether a correction is required or not. Determination of the necessity of the correction is no simple matter and requires detailed knowledge of the absorption contrasts of all contributing phases, along with their grain sizes in the sample being considered. Note that microabsorption is based upon the grain size of a material rather than the diffracting crystallite size. Application of an unnecessary microabsorption correction can result in severe underestimation of some phases and overestimation of the remaining phases in the sample (especially if methods are used which sum the total content to $100 \mathrm{wt} \%$ ). Two participants (designated $D$ and $E$ ) have investigated extensively the effects of microabsorption correction on sample 1. Both have used Rietveld refinement methods incorporating the Brindley correction model for microabsorption (Brindley, 1945).

Participant $D$ has expressed doubts about the particle sizes issued by the CPD and has gone to some lengths to recalculate them using the method of Scherrer (1918) based on observed peak widths. The results of participant $D$ using the Scherrer equation yield crystallite sizes of $0.511 \mu \mathrm{m}$ for corundum, $0.792 \mu \mathrm{m}$ for fluorite and $0.704 \mu \mathrm{m}$ for zincite. Participant $D$ also states that although there is uncertainty about the absolute scale of the crystallite sizes returned from the Scherrer equation, there is little doubt that the corundum has a diffracting size smaller than the other two components.

These results are in contrast with the CPD-supplied values of $28 \mu \mathrm{m}$ for corundum, $3.6 \mu \mathrm{m}$ for fluorite and $1.4 \mu \mathrm{m}$ for zincite. However, the sizes supplied by the CPD refer to particle size rather than crystallite size as calculated by the
Scherrer equation. It is most likely that the large particles of corundum are not single crystals but are composed of many smaller crystallites. However, it is the particle size rather than the crystallite size that needs to be considered when applying a microabsorption correction. To alleviate any doubt regarding the particle size, the authors have repeated the sizing of the individual materials using the same technique as originally used (Malvern Mastersizer laser sizing instrument). In addition, the sizing was carried out on sample $1 g$ to examine the possibility of particle size reduction or deagglomeration during the mixing process. The results of these additional measurements confirm particle sizes originally determined on the pure phase materials and supplied by the CPD. In fact, the results of particle size analysis of sample $1 g$ show three distinct peaks representing the zincite, fluorite and corundum particles.

Participant $D$ has carried out four replicate refinements, each on CPD-supplied data and on the participant-collected X-ray data. Each refinement has been conducted using the Siroquant program (Taylor, 1991) incorporating a different suite of crystallite sizes for the Brindley correction, as shown in Table 16. The approximate magnitude of the calculated correction factor $(\tau)$ is also given.

The sizes used in replicate 1 have been based upon the ratio of those determined using the Scherrer equation but scaled up according to the CPD-specified values. Replicate 2 represents the uncorrected refinement, replicate 3 represents that using the sizes derived from the Scherrer calculation, and replicate 4 represents the sizes supplied by the CPD. Figs. 16 and 17 show the $\mathrm{AKLD}_{\text {sum }}$ values for CPD-supplied and participantcollected data, respectively, according to participant $D$. Fig. 16 shows that for samples $1 d$ to $1 h$ (i.e. those with medium to

Table 15

Values of the reference intensity ratios (RIR) reported by participants.

Included for reference are data from the ICDD which include both measured and calculated values, values from the NBS SRM674 data sheet, and values calculated by the authors using the $\mathrm{Cu} K \alpha$ wavelength with the $S R 5$ Rietveld analysis program. The measured values were determined by the authors using the same materials used to prepare the sample 1 mixtures. Each measured value is the mean of three determinations and the value in parentheses is the standard deviation of the mean.

\begin{tabular}{|c|c|c|c|c|c|c|}
\hline \multirow[b]{2}{*}{ Participant } & \multicolumn{2}{|l|}{ Corundum } & \multicolumn{2}{|l|}{ Fluorite } & \multicolumn{2}{|l|}{ Zincite } \\
\hline & Analysed wt \% & RIR & Analysed wt\% & RIR & Analysed wt \% & RIR \\
\hline$A$ & 31.14 & 1.00 & 36.88 & 3.50 & 32.00 & 4.50 \\
\hline C & 30.48 & 1.00 & 34.58 & 3.30 & 35.03 & 4.35 \\
\hline
\end{tabular}

\begin{tabular}{|c|c|c|c|c|c|c|}
\hline \multirow[b]{2}{*}{ Source } & \multicolumn{2}{|l|}{ Corundum } & \multicolumn{2}{|l|}{ Fluorite } & \multicolumn{2}{|l|}{ Zincite } \\
\hline & ICDD Card Number & RIR & ICDD Card Number & RIR & ICDD Card Number & RIR \\
\hline \multirow[t]{5}{*}{ ICDD } & $10-173$ & 1.00 & 4-0864 & 2.40 & $5-0664$ & 4.50 \\
\hline & $71-1126$ & 1.02 & 75-0097 & 3.84 & $75-1533$ & 5.37 \\
\hline & $75-0782$ & 2.17 & $77-2245$ & 4.00 & $75-0576$ & 5.53 \\
\hline & & & & & $76-0704$ & 5.42 \\
\hline & & & & & $79-2205$ & 5.41 \\
\hline Smith et al. (1987) & - & 1.00 & - & 3.30 & - & 5.70 \\
\hline SRM674 & - & - & - & - & - & 5.17 \\
\hline Calculated & - & 1.00 & - & 3.67 & - & 4.90 \\
\hline Measured & - & 1.00 & - & $3.82(0.026)$ & - & $4.93(0.007)$ \\
\hline
\end{tabular}


Table 16

Particle sizes used by participant $D$ and the associated Brindley correction factors $(\tau)$.

\begin{tabular}{lrlllllll}
\hline & \multicolumn{2}{l}{ Corundum } & & \multicolumn{2}{l}{ Fluorite } & & \multicolumn{2}{l}{ Zincite } \\
\cline { 2 - 3 } Replicate number & $\begin{array}{l}\text { Size } \\
(\mu \mathrm{m})\end{array}$ & $\tau$ & & $\begin{array}{l}\text { Size } \\
(\mu \mathrm{m})\end{array}$ & $\tau$ & & $\begin{array}{l}\text { Size } \\
(\mu \mathrm{m})\end{array}$ & $\tau$ \\
\hline 1 & 8.4 & 1.076 & 13.0 & 0.942 & & 11.6 & 0.968 \\
2 & $<0.1$ & 1.000 & $<0.1$ & 1.000 & $<0.1$ & 1.000 \\
3 & 0.5 & 1.004 & & 0.8 & 0.996 & & 0.7 & 0.998 \\
4 & 28.0 & 1.290 & & 3.6 & 0.985 & & 1.4 & 0.997 \\
\hline
\end{tabular}

\section{Table 17}

Schedule of analyses used by participant $E$ for evaluating microabsorption correction.

\begin{tabular}{llll}
\hline $\begin{array}{l}\text { Replicate } \\
\text { number }\end{array}$ & $\begin{array}{l}\text { Analytical } \\
\text { method }\end{array}$ & $\begin{array}{l}\text { Data } \\
\text { collection }\end{array}$ & $\begin{array}{l}\text { Brindley correction } \\
\text { (particle sizes as } \\
\text { supplied by CPD) }\end{array}$ \\
\hline CPD-supplied data & & \\
1 & Siroquant & - & No \\
2 & Siroquant & - & Yes \\
3 & SR5 & - & No \\
4 & SR5 & - & Yes \\
Participant-collected data & & \\
1 & Siroquant & Automatic divergence slits & No \\
2 & Siroquant & Automatic divergence slits & Yes \\
3 & SR5 & Automatic divergence slits & No \\
4 & SR5 & Automatic divergence slits & Yes \\
5 & Siroquant & Fixed divergence slits & No \\
6 & Siroquant & Fixed divergence slits & Yes \\
7 & SR5 & Fixed divergence slits & No \\
8 & SR5 & Fixed divergence slits & Yes \\
\hline
\end{tabular}

\section{Table 18}

Weighed values for sample $1 g$ and values determined by participant $E$ using the SR5 Rietveld program with and without Brindley correction for the effects of microabsorption.

The particle sizes used are those supplied by the CPD.

\begin{tabular}{llll}
\hline & Corundum & Fluorite & Zincite \\
\hline Weighed & 31.37 & 34.42 & 34.21 \\
No correction & 32.8 & 35.6 & 31.6 \\
Brindley correction & 24.6 & 40.3 & 35.1 \\
\hline
\end{tabular}

\section{Table 19}

Spread in the relative bias at various levels of phase concentration averaged over all participants and all phases - CPD-supplied data.

The 25/75 column represents only those values which fall within the 25 th and 75th percentiles.

\begin{tabular}{lll}
\hline \multirow{2}{*}{$\begin{array}{l}\text { Approximate concentration } \\
\text { level (wt \%) }\end{array}$} & \multicolumn{2}{l}{ Absolute relative bias spread (\%) } \\
\cline { 3 - 3 } & All results & $25 / 75$ results only \\
\hline 5 & $9-26$ & $3-14$ \\
15 & $6-24$ & $3-13$ \\
30 & $6-12$ & $2-9$ \\
50 & $5-9$ & $1-5$ \\
95 & $3-4$ & $1-3$ \\
\hline
\end{tabular}

major amounts of each phase present), the correction based upon the sizes supplied by the CPD consistently provides the poorest analyses. This is also apparent for participantcollected data (Fig. 17). What is not obvious is any significant and systematic improvement in analysis with any of the other replicates.

Participant $E$ has carried out four replicate analyses on the CPD-supplied data and eight on two in-house-collected X-ray data sets for each sample, as given in Table 17.

Figs. 18, 19 and 20 show the AKLD $_{\text {sum }}$ values for CPDsupplied and participant-collected data (automatic and fixed divergence slits), respectively, according to participant $E$. For CPD-supplied data, the analyses performed with the SR5 Rietveld program [a local modification of the LHPM program of Hill \& Howard (1986)] without correction for microabsorption are significantly better than those with correction and those performed using Siroquant (Taylor, 1991) with and without correction. However, if the Siroquant analyses of participant $E$ are compared with those performed by participant $D$ (Fig. 16), it can be seen that they are significantly worse and may actually be a reflection of differing refinement strategies between participants $D$ and $E$ rather than the potential of either program. For participant-collected data, with automatic and fixed divergence slits, similar results are apparent (Figs. 19 and 20).

The magnitudes of the Brindley correction factors, $\tau$, used by participant $E$ are similar to those given in Table 16 (replicate 4). It can be seen that for corundum, sized as $28 \mu \mathrm{m}$, the factor of 1.290 has a dramatic effect on the reported QPA. Table 18 shows the weighed values for sample $1 g$ and the values determined by participant $E$ using $S R 5$ with and without Brindley correction.

It is clear from these results that the Brindley method does not adequately model the correction for microabsorption required for these samples. Even though the particle sizes have been confirmed, the Brindley model generates a correction factor that is much larger than is required for this phase system.

\subsection{Operator error during analysis}

A large number of results deviated significantly from the weighed values, largely due to inappropriate use of the analysis method by the operator rather than any systematic problem in data collection or analytical strategies. Given the degree of complexity of the Rietveld method and the fact that the majority of participants have elected to use this method, it is inevitable that many of the errors in the analyses arise from misuse of the various Rietveld programs. An incomplete list of examples follows.

(a) Several participants set the overall phase thermal parameters plus the individual atom thermal parameters to zero for all phases and did not refine them during analysis. This has the effect of misrepresenting the peak intensities with compensation in the phase scale factor. The errors are then propagated to the quantitative analysis. 


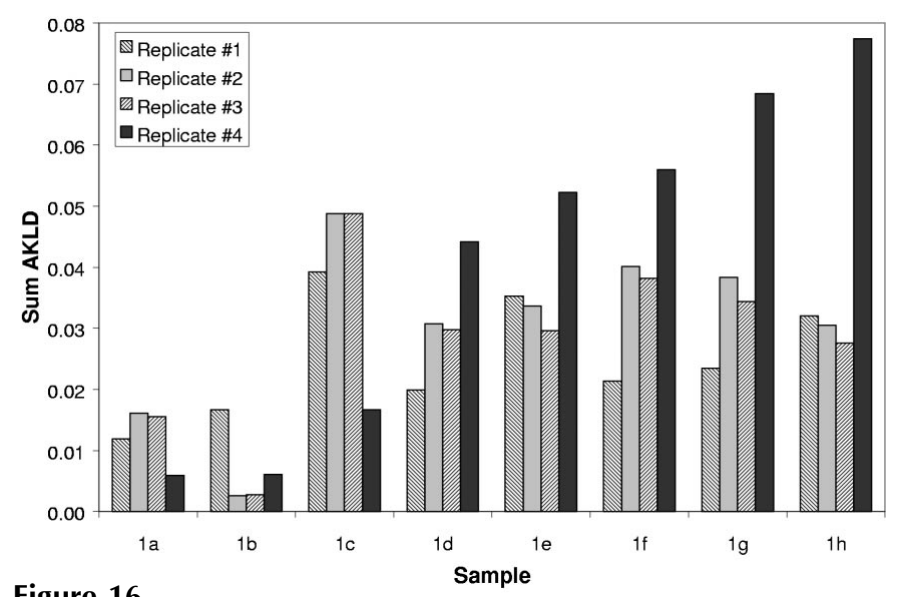

Figure 16

The effect of microabsorption correction on $\mathrm{AKLD}_{\text {sum }}$ for CPD-supplied data according to participant $D$. The replicates relate to the use of different particle sizes in the Brindley microabsorption correction function. Replicate 1 uses particle sizes derived by the participant, replicate 2 effectively applies no correction, replicate 3 uses the crystallite sizes derived from the Scherrer equation, and replicate 4 uses the particle sizes supplied by the CPD.

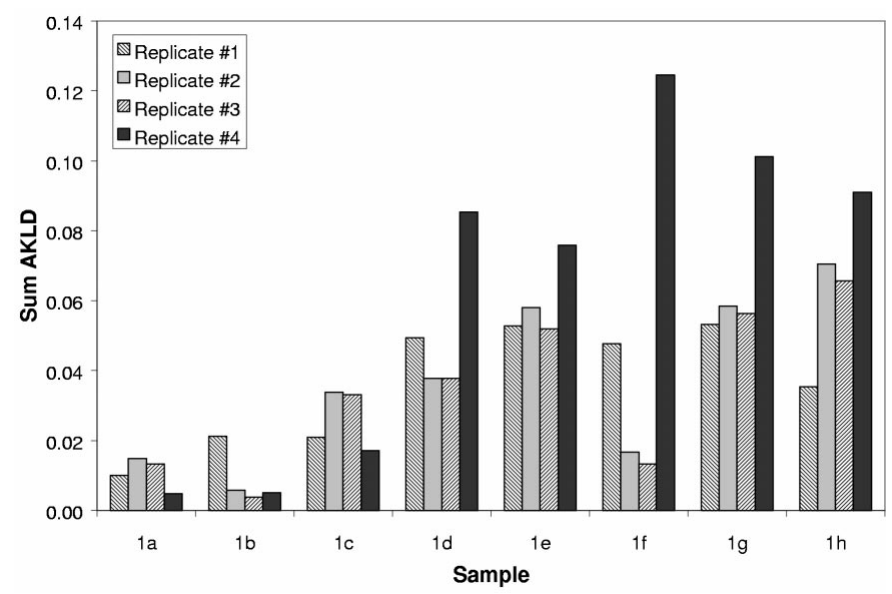

Figure 17

The effect of microabsorption correction on $\mathrm{AKLD}_{\text {sum }}$ for participantcollected data according to participant $D$. Explanation of the difference between replicates is given in Fig. 16.

(b) At least two participants entered the zincite structure with the $\mathrm{Zn}$ atom at $(1 / 3,2 / 3,0.0500)$ rather than at the correct value of $(1 / 3,2 / 3,0.5000)$. The effect of this error on sample $1 g$ (checked by the authors using the SR5 Rietveld program) was to reduce the reported zincite analysis to $26.2 \mathrm{wt} \%$, which is somewhat less than the weighed amount of $34.2 \mathrm{wt} \%$. While the quantitative analysis might have been accepted as reasonable, the high Rietveld refinement $R$ factors $\left(R_{\mathrm{wp}}=\right.$ $44.1 \%$ ) should have indicated to the operators that there were residual errors in the analysis.

(c) For sample $1 g$ (CPD-supplied data), one participant returned values for corundum, fluorite and zincite of 9.6, 80.4 and $10.0 \mathrm{wt} \%$, respectively, compared with the weighed values of $31.3,34.4$ and $34.2 \mathrm{wt} \%$. The participant entered the space group for fluorite as $\mathrm{Fm} 3 / \mathrm{m}$ instead of $\mathrm{Fm} 3 \mathrm{~m}$. Rather than rejecting the (non-existent) space-group setting, the Rietveld

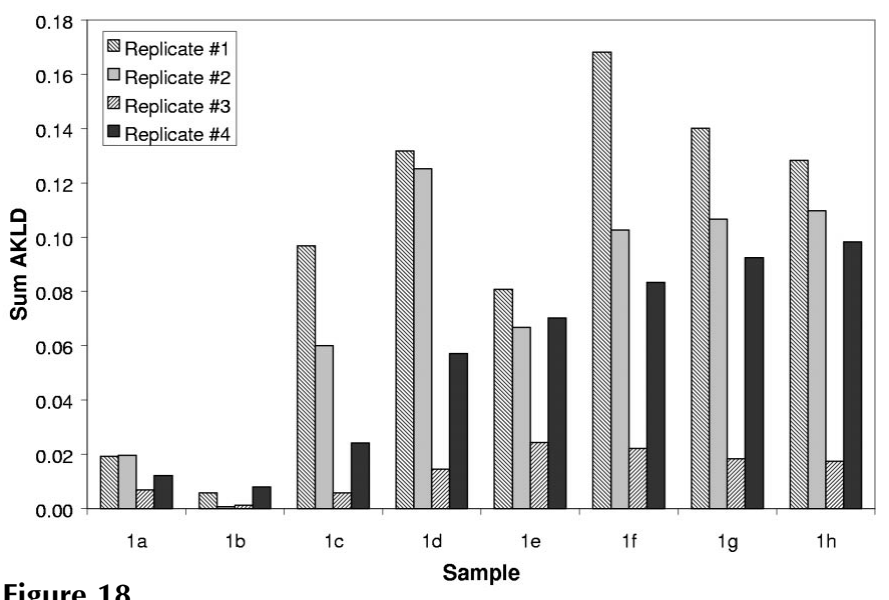

Figure 18

The effect of microabsorption correction on $\mathrm{AKLD}_{\text {sum }}$ for CPD-supplied data according to participant $E$. The replicates relate to the use of different Rietveld analysis programs with and without application of the Brindley microabsorption correction function. Replicate 1 uses Siroquant without correction, replicate 2 uses Siroquant with correction, replicate 3 uses $S R 5$ without correction, and replicate 4 uses $S R 5$ with correction.

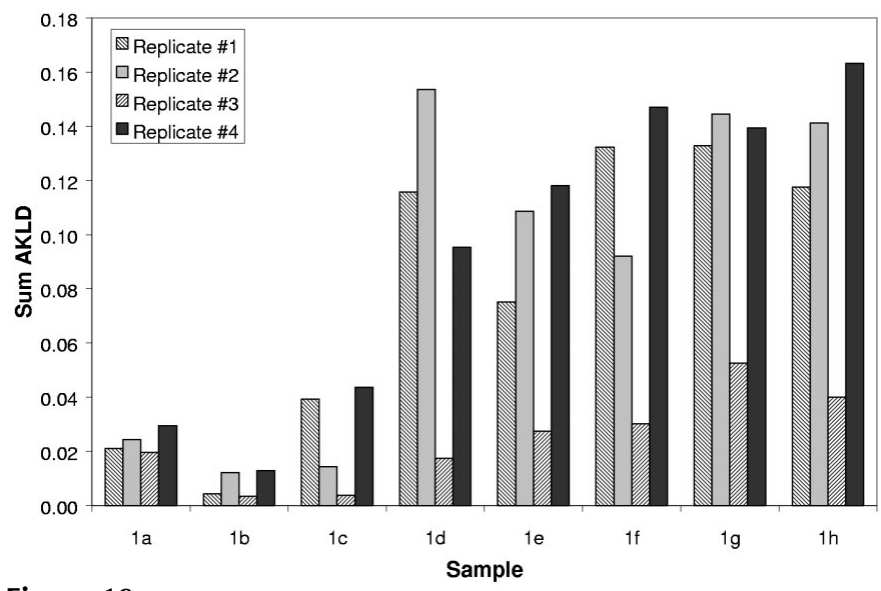

Figure 19

The effect of microabsorption correction on $\mathrm{AKLD}_{\text {sum }}$ for participantcollected data (automatic divergence slit) according to participant $E$. Explanation of the difference between replicates is given in Fig. 18.

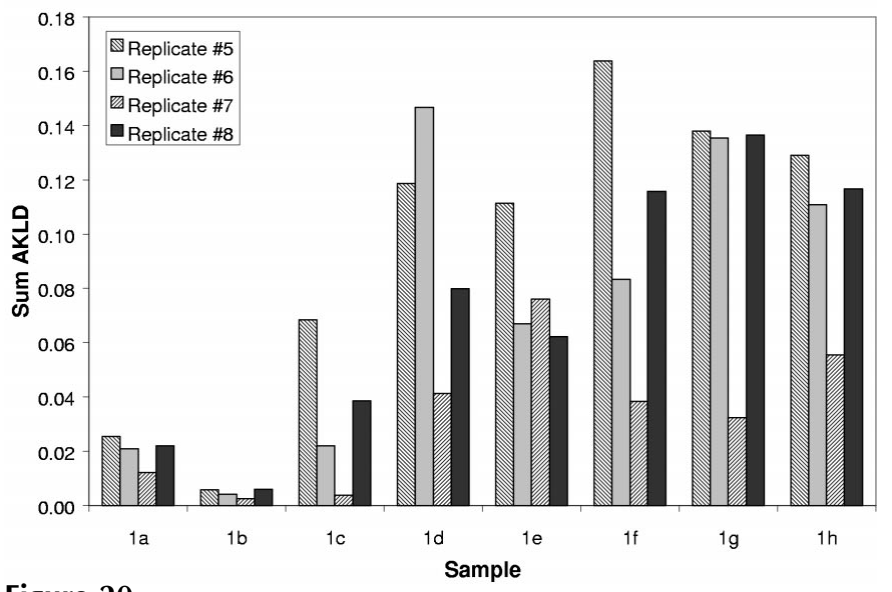

Figure 20

$\mathrm{AKLD}_{\text {sum }}$ for participant-collected data (fixed divergence slit) according to participant $E$. Explanation of the difference between replicates is given in Fig. 18. 


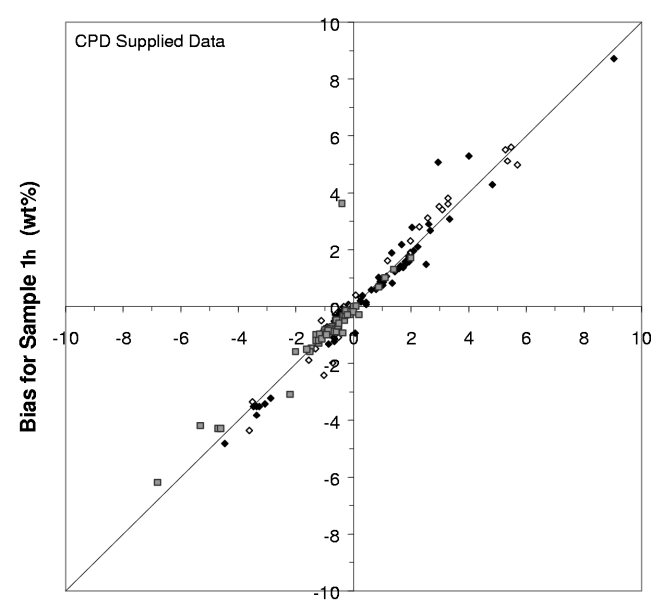

Bias for Sample 1g (wt\%)

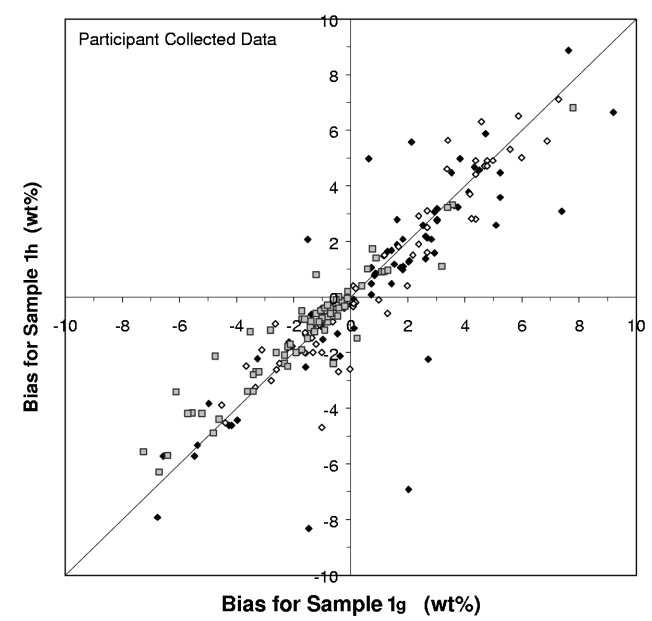

Figure 21

Summary of the comparison of precision and accuracy for samples $1 g$ and $1 h$, for each phase and for all returns. Corundum is represented by filled diamonds, fluorite by open diamonds and zincite by grey squares.

program assigned reflection multiplicities based on the spacegroup symbols provided. The Rietveld scale factor then refined to an incorrect value, resulting in a large error in the derived phase abundances.

(d) Several participants simply omitted corundum from the analysis of sample $1 a$ (CPD-supplied and participant-collected data) in spite of being informed that the sample 1 mixtures were composed of three phases. Even though corundum is present at only $1.2 \mathrm{wt} \%$, its peaks are still apparent in the observed diffraction patterns. An error such as this may be due to a lack of care in the initial examination of the data prior to the quantitative analysis.

(e) One participant entered all site occupation factors (SOF) as the total number of atoms in the unit cell rather than as the ratio of the site multiplicity to the multiplicity for the general site. For example, in corundum, the Al SOF was entered as 12 rather than 0.33333 as required by the program. Even though the relative calculated peak intensities are
Table 20

Spread in the relative bias at various levels of phase concentration averaged over all participants and all phases - participant-collected data.

The 25/75 column represents only those values which fall within the 25th and 75 th percentiles.

\begin{tabular}{lll}
\hline \multirow{2}{*}{$\begin{array}{l}\text { Approximate concentration } \\
\text { level (wt\%) }\end{array}$} & \multicolumn{2}{l}{ Absolute relative bias spread (\%) } \\
\cline { 2 - 3 } & All results & $25 / 75$ results only \\
\hline 1 & $14-49$ & $5-15$ \\
5 & $9-20$ & $3-9$ \\
15 & $7-13$ & $4-6$ \\
30 & $5.5-8.5$ & $2-4$ \\
50 & $4.2-4.8$ & $1.5-2$ \\
95 & $0.5-1.2$ & $0.2-0.6$ \\
\hline
\end{tabular}

correct, their overall magnitude is much larger than expected. This causes the Rietveld program to generate an incorrect scale factor as well as calculating an incorrect ZMV (where $\mathrm{ZM}$ is the mass of the contents of the unit cell and $\mathrm{V}$ is the unit-cell volume) (Hill, 1983; Hill \& Howard, 1987) used to convert the scale factor to phase abundance. Calculation of crystal density and comparison with experimental density would have revealed this error. For example, the crystal density of corundum is $3.99 \mathrm{~g} \mathrm{~cm}^{-3}$, but is calculated as $143.7 \mathrm{~g} \mathrm{~cm}^{-3}$ if the SOF reported above is used. Such a calculation could be incorporated into Rietveld codes as a standard feature.

( $f$ ) Again for sample $1 g$ (CPD-supplied data), one participant returned values for corundum, fluorite and zincite of 14.6, 31.1 and $59.3 \mathrm{wt} \%$, respectively. These values resulted from a simple misreading of the Rietveld program output file. The correct values (appearing later in the output file) of 31.6, 34.3 and $34.1 \mathrm{wt} \%$ agree well with the weighed values of $31.3,34.4$ and $34.2 \mathrm{wt} \%$.

There were a number of other minor errors in the Rietveld refinement strategy employed by many participants. While not producing gross errors in the QPA, they still have the potential to affect the phase abundances. Attention to the details of the refinement methodology should ensure minimal deviation from the 'true' values. Some examples of these errors follow.

(i) Peak widths were set to unrealistically small values and not refined during the analysis. Residual errors in fitting the peaks have the potential to affect the scale factors and hence the QPA. This indicates that the operators may not be examining the graphical output for residual errors.

(ii) The unit-cell dimensions were fixed at the values supplied by the CPD. The supplied values were only indicative and were to be used as a starting point by participants. While the Rietveld programs will still generate peaks in approximately correct positions, any residual errors in peak position have the potential to affect the scale factors and hence the QPA.

(iii) Several participants failed to apply appropriate constraints in the refinement of the unit-cell dimensions. For the hexagonal structure types (corundum and zincite), the value of $\gamma$ was allowed to deviate from $120^{\circ}$. While the 
difference was not significant and did not affect the QPA, it indicates a lack of understanding of the operating requirements of the various Rietveld programs.

(iv) Many participants have returned analyses based on incomplete Rietveld refinement. This is evidenced by the high values of the weighted profile $R$ factor $R_{\mathrm{wp}}$ (up to $50 \%$ ) and the Bragg $R$ factors $R_{B}$ (up to $33 \%$ ). At this level, the misfit between observed and calculated intensity should be easily observed in the graphical output and will certainly be sufficient to affect the scale factors and hence the QPA.

Many of these errors by participants indicate either a lack of crystallographic expertise, or understanding of program operation and/or interpretation of the output. There is a tendency, in the field of QPA, to treat some analysis programs as a 'black box' with data and (sometimes incomplete) analysis conditions being fed in and results extracted without a detailed understanding of the analysis mechanism. However, many current Rietveld-based programs do not contain the checks and balances required to eliminate the possibility of mistakes occurring, such as those given above. Most Rietveld programs currently assume a certain level of knowledge on behalf of the operator. Continued education of Rietveld users through regular workshops and training publications (McCusker et al., 1999) should assist in avoiding this type of error.

\subsection{Precision and accuracy}

Discussion of the problems associated with the estimation of error in the phase abundances is limited by the fact that users have not specified the methods of error calculation employed. In some instances, quoted errors in the QPA have been derived from the errors in the Rietveld scale factors. It must be remembered that the errors calculated by Rietveld software consider only the errors in precision in the calculation and are not necessarily related to the accuracy of the determination. In most cases, the Rietveld estimated standard deviations (e.s.d.'s) represent only a small component of the 'true' analytical error.

Tables 19 and 20 show the average relative biases for all phase abundances derived using CPD-supplied and participant-collected data, respectively, and indicate the spread of results received during the round robin. It is worth noting that individual participants have performed better than the spread would indicate. In addition, the accuracy of analysis depends in part on the phase being analysed. For example, zincite is the heaviest scatterer of X-rays (on which the bulk of the returns are based) and is generally better determined at lower levels of concentration than either corundum or fluorite.

Tables 19 and 20 report the range of relative biases at each approximate concentration level examined in this survey. Results are reported for all returns and for those which fall between the 25th and 75th percentiles. The 25th and 75th percentiles represent the values below which 25 and $75 \%$ of all returns fall, respectively. Considering only the values that fall between these levels takes into account only $50 \%$ of the returns. If the returns approximate a normal distribution, data within the 25/75 limits represent one e.s.d. of the entire group.

Table 19 shows that the entire community achieved an absolute accuracy of $9-26 \%$ relative at the approximately $1 \mathrm{wt} \%$ level when analysing CPD-supplied data. This improves dramatically to $3-14 \%$ relative if only the values falling within the $25 / 75$ band (i.e. $50 \%$ of the community) are considered. The level of accuracy achievable at about the $95 \mathrm{wt} \%$ level is of the order of $0.4-1.5 \%$ relative for all results and $0.1-0.8 \%$ relative for the $25 / 75$ results. Table 20 shows that the corresponding figures for the participant-collected data are considerably worse for all data at the lower concentration limits, i.e. at about the $1 \mathrm{wt} \%$ level. However, at higher concentration levels, the results are comparable with those obtained by analysing the CPD-supplied data. The results from the 25/75 band of the participant-collected data are comparable with the CPD-supplied data at all concentration levels.

Fig. 21 summarizes the reproducibility estimates based on samples $1 g$ and $1 h$, for each phase and for all returns. The major features of this figure can be interpreted as follows.

(a) Values at the centre of the plot: the participant is able to replicate their result with little apparent bias (i.e. they are both precise and accurate).

(b) Values on or near the 1:1 line, but in the top-right quadrant: the participant is able to replicate their results, but there is a systematic bias in their method such that both values are overestimated (i.e. they are precise but inaccurate).

(c) Values on or near the 1:1 line, but in the bottom-left quadrant: the participant is able to replicate their results, but there is a systematic bias in their method such that both values are underestimated (i.e. they are precise but inaccurate).

(d) Values off the 1:1 line: the participant is unable to repeat their analyses (i.e. they are imprecise and one or both determinations may be inaccurate).

The most precise analyses lie along the ' $1: 1$ ' line, i.e. when the bias in estimation of phase abundance in $1 g$ is the same as that in $1 h$. A participant may show excellent reproducibility but be severely inaccurate as far as absolute bias is concerned. In general, the accuracy of analysis of CPD-supplied data is similar to that of participant-collected data, but the precision, or reproducibility, of analysis based upon CPD-supplied data is better.

\subsection{Inappropriate laboratory $\mathrm{X}$-ray data collection regimes}

It is beyond the scope of this study to determine the problems with data collection regimes being employed throughout the community. However, it is evident from the returns that, in general, QPA, especially at the lower concentration levels, has been more accurately executed on Xray data provided by the CPD than on participant-collected laboratory X-ray data. This suggests that some attention needs be paid to the factors affecting the collection of diffraction data, including instrument configuration and data collection strategies. 


\section{Conclusions}

The methods employed for data collection and QPA have been documented and assessed with respect to phase abundance determination in a simple three-phase mixture. Since the majority of returns were derived from X-ray data analysed using the Rietveld method, this combination of instrumentation and methodology is well represented in the statistical analysis of results. The levels of accuracy and precision of QPA have been determined using various frames of reference for assessment of the community's performance, i.e., comparing returns on the basis of data collection method, analytical technique employed, mathematical corrections applied and reproducibility. This has revealed certain common problems that are currently restricting the success of QPA via diffraction methods. The lower limits of detection of the methods have been assessed qualitatively through the presence of phases of varying scattering power and absorption at approximately the $1 \mathrm{wt} \%$ level.

No formal procedures for QPA have been recommended, but several detailed recommendations are made regarding the most pertinent problems encountered in the survey. It is hoped that by addressing the individual problems in this way, a general improvement in QPA procedures will result.

The following points summarize the various problems encountered.

(i) There are significant difficulties associated with the use of RIR values for QPA. A wide range of the returned analyses were dependent, at least partially, upon the RIR value chosen. There are considerable discrepancies between different published RIR values and there are probably further differences in values derived by operators at the time of analysis. It is not possible to comment further on this as not all participants who employed this method supplied the RIR values used. However, from the values that were supplied, it is apparent that this is an area which needs attention from the community if the method is to continue to be used.

(ii) One of greatest problems in QPA by X-ray diffraction is correction for the effects of microabsorption. For the analyst encountering a new sample, it is difficult to determine whether a correction for microabsorption is required without first obtaining additional information. This may consist of determining the particle sizes by laser-sizing or scanning electron microscopy observations, but even when these parameters are known, the best form of correction may still be unclear. The most frequently applied correction in this study is based on the Brindley model. This requires detailed knowledge of the absorption contrast between, and particle sizes of, the different phases within the sample mix. Since the model assumes that each phase is composed of spherical particles of uniform size, its application may be unrealistic in a 'real world' situation where a range of particle sizes and shapes can be encountered for each phase. The problem of knowing when a correction is necessary is a very real one and many of the returns discussed here suffered severely from the application of excessively large correction factors. Incorrect values for correction may also arise from participants not working within the limits of the Brindley model. These may require additional specification and clarification for users of the model.

(iii) Many of the errors in the analyses can be attributed to the use of Rietveld analysis programs by operators with insufficient crystallographic and/or chemical knowledge. This results in errors derived from $(a)$ the use of incorrect space groups, $(b)$ the use of incorrect site occupation factors, $(c)$ the acceptance of physically unrealistic parameters for the phase, (d) the incorrect interpretation or simple misreading of output files, and $(e)$ the acceptance of results based on incomplete refinement.

(iv) One area of quantitative analysis that needs urgent attention is the calculation of error estimates for the results. Error estimates were generally reported by ILS and internalstandards users, by some Rietveld and RIR users, and not at all by full-pattern-match users. The errors calculated by Rietveld analysis programs are concerned only with the mathematical precision of the calculation itself and do not necessarily reflect the accuracy of the result.

(v) The community as a whole has achieved more reproducible results with the CPD-supplied data than with participant-collected data. This implies that there may be some problems associated with the collection of data that must be addressed if precision in quantitative analysis is to improve. The results achieved using both CPD-supplied and participant-collected data are, on the whole, encouraging and indicate that quite high levels of accuracy, down to approximately the $1 \mathrm{wt} \%$ level, are possible using laboratory X-ray equipment. This is important as laboratory-based X-ray methods are by far the most common and easily accessed. This finding provides a benchmark for quantitative analysis of a simple system under the best possible conditions. More complex systems, with their associated problems of phase identification, peak shifts through chemical substitution, increased microabsorption, preferred-orientation problems, and excessive peak overlap, can only reduce the levels of accuracy and precision that can be attained.

Limited quantities of some of the samples distributed during the round robin are still available for individuals who wish to verify their own data collection and analysis techniques. Samples can be obtained by contacting the first author.

\section{Recommendations}

\subsection{Reference intensity ratios}

The derivation and use of reference intensity ratios has been well examined (Hubbard \& Smith, 1977; Hubbard \& Snyder, 1988; Davis \& Smith, 1988; Davis et al., 1989). However, there seems to be a need for greater education of the community in their usage. This may be assisted by revisiting the tabulations of existing RIR values and indicating more clearly the method of derivation in each case (peak area, peak height, whole-pattern). Clarity in the derivation may assist users to choose the RIR most appropriate to their situation. It may be that in some cases it is necessary for an appropriate RIR to be derived at the time of the experiment in 
order to account for such variables as instrumental configuration and differing crystallinities of component phases. Continuing education of users in the methodology required to derive these values may still be required.

\subsection{Rietveld refinement}

The main issue in the use of the Rietveld method for QPA is also the education of operators. In many cases, there is a clear lack of crystallographic understanding as well as problems in the operation of software. The need to refine 'non-crystallographic' parameters (e.g. peak width and shape) and their impact on QPA also appears not to be clearly understood. The interpretation of the Rietveld output files has caused confusion for some participants, especially in knowing when the refinement is complete (evidenced in acceptable values of the various measures of fit such as $R_{\mathrm{wp}}$ and $R_{B}$ ).

Many of these problems have already been discussed by Hill (1992) and Hill \& Cranswick (1994) in their round-robin studies on structure refinement. They highlighted several important issues affecting accuracy in Rietveld refinement, including (i) refinement strategy, (ii) the use of poor profile models, (iii) termination of refinement before convergence is achieved, and (iv) refinement of parameters not supported by the data. Since there is considerable overlap with the findings of this work, it would appear that the recommendations of the previous studies have not been as widely accepted by the diffraction community as is desirable.

It may also be possible to build more 'intelligence' into Rietveld codes and issue more warnings for potentially serious errors. For example, warnings may be provided for the following.

(a) Atom and overall thermal parameters which are physically unrealistic (negative, zero or large positive values).

(b) Non-standard space groups.

(c) Site occupation factors which are severely incorrect. These produce Rietveld scale factors which deviate significantly from the expected values, and, for those programs which perform the appropriate cycle-by-cycle calculations, high ZMV values and high calculated phase density. Such calculations could be included as a standard feature.

\subsection{Data collection}

The larger spread of results derived from participantcollected data compared with those from CPD-supplied data indicates that some of the variability arises from the manner in which the diffraction data are collected, especially at the lower concentration levels. Continuing education is required in the areas of instrumental configuration for powder diffraction and data collection strategies for QPA. Further discussion of these issues can be found in works by Young (1993) and Bish \& Post (1989).

\subsection{Microabsorption}

The mixtures comprising sample 1 required very little in the way of microabsorption correction in spite of the presence of large particles of corundum. Application of correction models, such as that defined by Brindley (the most commonly employed in this study), can result in severe overcorrection of the quantitative phase analyses. This was particularly apparent in samples $1 d$ to $1 h$, in which all three phases were present in significant amounts, and in the studies conducted by participants $D$ and $E$. This implies that the correction models in current use are not adequate and need to be revised as a matter of high priority.

\subsection{Error calculation}

The errors reported in this study covered a very wide range, indicating that there is little standardization in the methodology used to derive these values. Error estimates derived from Rietveld scale-factor e.s.d.'s do not represent the accuracy in QPA, but are often the only measure available to the analyst and are quoted accordingly. Further work is required on the methodology used to derive appropriate errors in QPA. Factors that need to be considered include the following.

(i) Mathematical precision in fitting the model to the data.

(ii) Effect of counting statistics.

(iii) Effect of phase abundance (i.e. higher phase concentration results in lower relative standard deviation).

(iv) Effect of scattering power of the phase in the case of Xray data (i.e. higher scattering power results in lower relative standard deviation).

To date, the errors calculated by Rietveld software consider only the first point (mathematical precision in fitting the model to the data) and yet are used to derive the absolute error in phase abundance by many operators.

The authors wish to acknowledge the following individuals for their contributions to the project: (i) Dr R. J. Hill, who, as past chairman of the IUCr Commission for Powder Diffraction (CPD), instigated the project, provided experimental design input and has provided on-going support for the round robin within CSIRO Minerals, (ii) Professor D. K. Smith for experimental design input at the start of the project, (iii) Dr R. J. Cernik for on-going support during his chairmanship of the CPD, and (iv) Professor Paolo Scardi for support as the current chairman of the CPD. Also, the financial support of the CPD is gratefully acknowledged. Finally, the authors would like to thank the many participants who have made this project possible. Many have obviously spent a great deal of time and effort in conducting their analyses and their submissions to the round robin have been very much appreciated. Only through the continued cooperation of the diffraction community will the field of quantitative phase analysis reach its full potential.

\section{References}

Bish, D. L. \& Post, J. E. (1989). Reviews in Mineralogy, Vol. 20, Modern Powder Diffraction. Washington: Mineralogical Society of America. (ISBN 0-939950-24-3.)

Brindley, G. W. (1945). Philos. Mag. 36, 347. 
Coelho, A. A., Madsen, I. C. \& Cheary, R. W. (1997). A New Rietveld Refinement Program Using a Fundamental Parameters Approach to Synthesizing Line Profiles, Abstract D1-PM2, Proceedings of the 20th Conference of the Society of Crystallographers in Australia, 2-5 April, Queenstown, New Zealand.

Davis, B. L. \& Smith, D. K. (1988). Powder Diffr. 3, 205208.

Davis, B. L., Smith, D. K. \& Holomany, M. A. (1989). Powder Diffr. 4, 201-205.

Hill, R. J. (1983). J. Power Sources, 9, 55-71.

Hill, R. J. (1992). J. Appl. Cryst. 25, 589-610.

Hill, R. J. \& Cranswick, L. M. D. (1994). J. Appl. Cryst. 27, 802-844.

Hill, R. J. \& Howard, C. J. (1986). A Computer Programme for Rietveld Analysis of Fixed-Wavelength X-ray and Neutron Powder Diffraction Patterns, AAEC (now ANSTO) Report AAEC/M112. Hill, R. J. \& Howard, C. J. (1987). J. Appl. Cryst. 20, 467-474.

Hubbard, C. R. \& Smith, D. K. (1977). Adv. X-ray Anal. 20, 27-39. Hubbard, C. R. \& Snyder, R. L. (1988). Powder Diffr. 3, 74-77.
ICDD (1999). The Powder Diffraction File, Database of the International Center for Diffraction Data, Sets 1-49 and 70-86, 12 Campus Boulevard, Newton Square, Pennsylvania 19073-3273, USA.

Knudsen, T. (1981). X-ray Spectrom. 10, 54-56.

Kullback, S. (1968). Information Theory and Statistics, pp. 1-11. New York: Dover.

McCusker, L. B., Von Dreele, R. B., Cox, D. E., Louer, D. \& Scardi, P. (1999). J. Appl. Cryst. 32, 36-50.

Rietveld, H. M. (1969). J. Appl. Cryst. 2, 65-71.

Sabine, T. M., Hunter, B. A., Sabine, W. R. \& Ball, C. J. (1998). J. Appl. Cryst. 31, 47-51.

Scherrer, P. (1918). Nachr. Ges. Wiss. Göttingen, Math.-Phys. Kl. Issue 2, 96-100.

Smith, D. K., Johnson, G. G., Scheible, A., Wims, A. M., Johnson, J. L. \& Ullmann, G. (1987). Powder Diffr. 2, 73-77.

Stalick, J. (1998). Personal communication.

Taylor, J. (1991). Powder Diffr. 6, 2-9.

Young, R. A. (1993). The Rietveld Method. Oxford University Press. (ISBN 0-19-8555776-6.) 